NASA Technical Memorandum 112194

\title{
Measurement of Air Flow Characteristics Using Seven-Hole Cone Probes
}

Timothy T. Takahashi, Ames Research Center, Moffett Field, California

May 1997

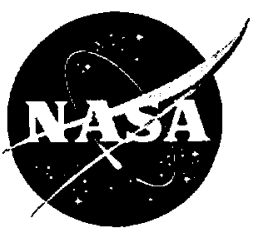

National Aeronautics and Space Administration

Ames Research Center

Moffett Field, California 94035-1000 



\section{Contents}

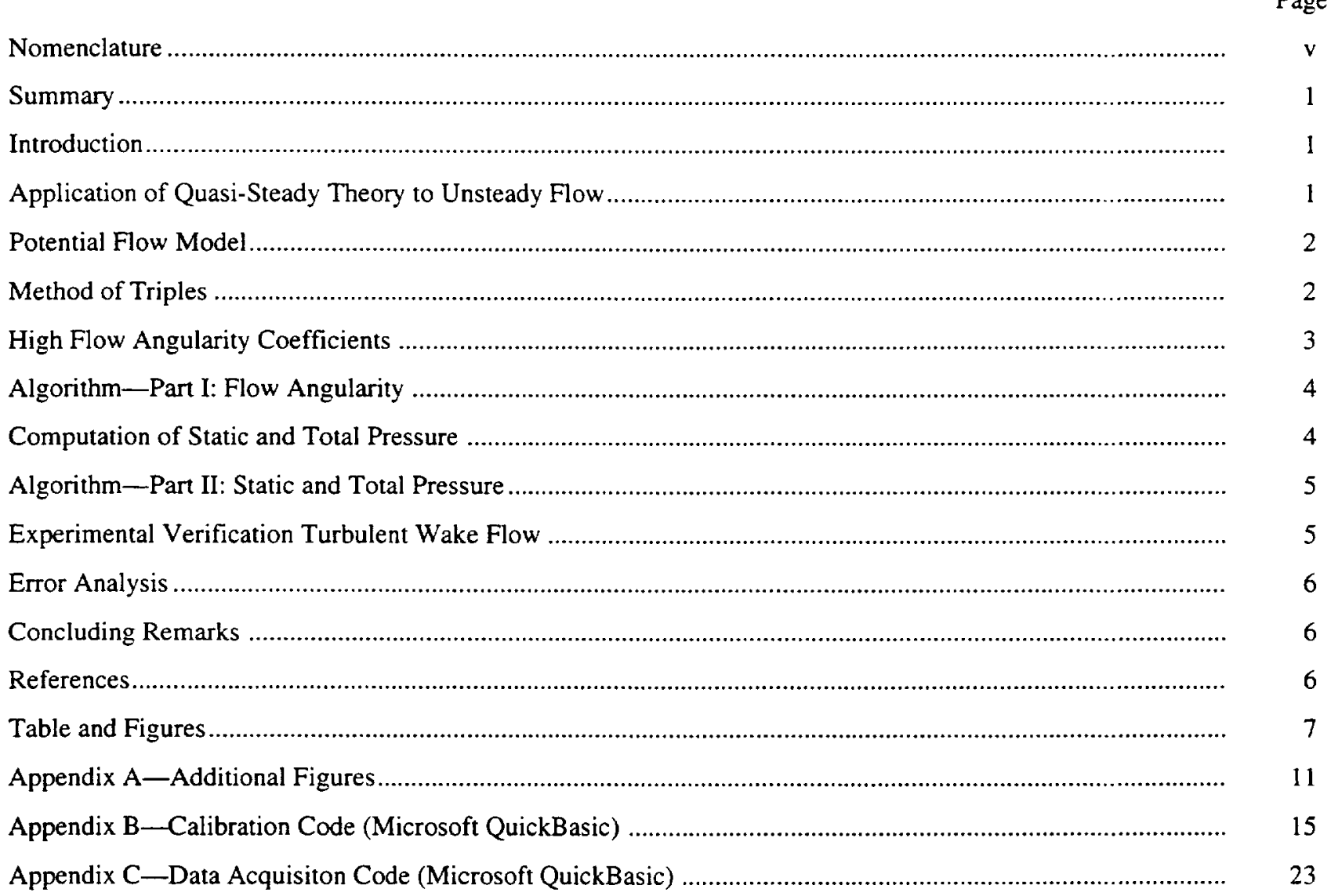





\begin{tabular}{|c|c|c|c|}
\hline \multicolumn{2}{|c|}{ Nomenclature } & \multirow{2}{*}{$\begin{array}{l}\overline{\mathrm{P}}_{\mathrm{s}} \\
\mathrm{P}_{\mathrm{s}}^{\prime}\end{array}$} & \multirow{2}{*}{$\begin{array}{l}\text { time-averaged static pressure } \\
\text { unsteady static pressure }\end{array}$} \\
\hline$C_{P}$ & dimensionless pressure coefficient & & \\
\hline \multirow[t]{2}{*}{$\mathrm{C}_{\mathrm{Ps}}$} & \multirow{2}{*}{$\begin{array}{l}\text { static pressure sensitive dimensionless } \\
\text { coefficient }\end{array}$} & $\mathrm{P}_{\mathrm{t}}$ & total pressure \\
\hline & & $\overline{\mathrm{P}}_{\mathrm{t}}$ & time-averaged total pressure \\
\hline $\mathrm{C}_{\mathrm{PT}}$ & $\begin{array}{l}\text { target pressure sensitive dimensionless } \\
\text { coefficient }\end{array}$ & $P_{t}^{\prime}$ & unsteady total pressure \\
\hline \multirow{3}{*}{$\begin{array}{l}\mathrm{C}_{\mathrm{Pt}} \\
\mathrm{C}_{\mathrm{Pta}}\end{array}$} & \multirow{2}{*}{ total pressure sensitive dimensionless coefficient } & $\mathrm{q}$ & dynamic pressure \\
\hline & & $\operatorname{Re}$ & Reynolds number \\
\hline & pressure coefficient & $\mathrm{U}$ & Downstream velocity \\
\hline $\mathrm{CPtb}_{\mathrm{Ptb}}$ & pressure coefficient & $\overline{\mathrm{U}}$ & time-averaged downstream velocity \\
\hline $\mathrm{CPtc}_{\mathrm{Ptc}}$ & pressure coefficient & $\mathrm{U}^{\prime}$ & unsteadv downstram velocity \\
\hline $\mathrm{C}_{\mathrm{P} \alpha}$ & $\begin{array}{l}\text { pitch sensitive dimensionless pressure } \\
\text { coefficient }\end{array}$ & $\mathrm{x}$ & wake survey downstream distance \\
\hline $\mathrm{C}_{\mathrm{P} \beta}$ & yaw sensitive dimensionless pressure coefficient & $\alpha$ & flow angularity "pitch" \\
\hline $\mathrm{d}$ & diameter of circular cylinder (wake survey) & $\beta$ & flow angularity "yaw" \\
\hline $\mathrm{i}$ & index variable (port number) & $\gamma$ & Empirical coefficient (potential flow) \\
\hline $\mathrm{K}$ & empirically determined coefficient & $\varepsilon$ & empirical coefficient (potential flow) \\
\hline \multirow[t]{2}{*}{$\overline{\mathrm{P}}$} & \multirow{2}{*}{$\begin{array}{l}\text { instantaneous mean pressure of off-axis pressure } \\
\text { ports }\end{array}$} & $\Theta$ & included angle of incidence (potential flow) \\
\hline & & $\lambda$ & probe port "cone angle" \\
\hline $\mathbf{P}_{\mathbf{i}}$ & pressure at the $i$ th port of the seven-hole probe & $\Pi$ & dimensionless flow angularity coefficient \\
\hline$P_{S}$ & static pressure & $\phi$ & probe port "clock angle" \\
\hline
\end{tabular}





\title{
Measurement of Air Flow Characteristics Using Seven-Hole Cone Probes
}

\author{
TIMOTHY T. TAKAHASHI \\ Ames Research Center
}

\section{Summary}

The motivation for this work has been the development of a wake survey system. A seven-hole probe can measure the distribution of static pressure, total pressure, and flow angularity in a wind tunnel environment. The author describes the development of a simple, very efficient algorithm to compute flow properties from probe tip pressures. Its accuracy and applicability to unsteady, turbulent flow are discussed.

\section{Introduction}

For many years, multi-hole pressure probes have been used to measure flow angularity (refs. 1 and 2). The basic principle of operation is that a flow of given angularity and velocity at a given static pressure determines a unique pressure pattern across the various ports. The seven-hole probe has a specific construction advantage: six tubes of equal diameter fit exactly around a central tube of the same diameter. The geometry of the seven-hole probe is shown in figure 1 . The resulting system is overdetermined; there are more observations than states. The seven measured pressures determine the four output parameters: static pressure $P_{S}$, total pressure $P_{t}$, and flow angularity $\alpha$ and $\beta$.

In general, multi-hole pitot probes are used under the assumption that the flow of interest is steady. Great care is taken in the calibration process to ensure that the calibration flow is uniform and free from turbulence. However, real flows, particularly wake flows, are far from steady. The response of the probe to unsteady flow must be documented.

The decomposition algorithm, which reduces the seven pressures into the four air flow parameters, may be built using an ad hoc approach, an analytical flow model, or a mixture of theoretical and empirical rationale. This paper will describe the search for a procedure which combines good data quality with extreme processing efficiency.

\footnotetext{
* This work was performed while the author held a National Research Council-NASA Ames Research Associateship.
}

Thanks to Pat Moriarty, Stanford University, for the hotfilm anemometry data and to Tony Whitmore, Dryden Flight Research Center, for inspiration in the "triples" derivation.

\section{Application of Quasi-Steady Theory to Unsteady Flow}

There is a need to understand the applicability of any steady-state analysis, calibration, or computational procedure to the unsteady, highly turbulent flow often found in wake flow. In the past, many authors addressed this topic. A brief survey of some relevant publications is given here. Together, they describe issues important in the design of seven-hole probes.

Goldstein (ref. 3 ) postulated that the response of a total head probe in a turbulent stream, $P_{t}$, is a measurement of mean total pressure $\bar{P}_{t}$ plus a steady state contribution due to the unsteady velocity field:

$$
P_{t}=\overline{P_{t}}+P_{t}^{\prime}=P_{S} 1 / 2 \rho \bar{U}^{2}+1 / 2 \rho \overline{U^{\prime 2}}
$$

In other words, Goldstein considered static pressure a mean flow property.

Jenkins (ref. 4) examined a free jet with a pitot tube equipped with a wide-bandwidth transducer. He correlated the unsteady pitot pressures with cross-wire hot-film velocimetry measurements. He found that the unsteady velocities as expressed by:

$$
\mathrm{U}^{\prime}=(\overline{\mathrm{U}} / 2 \mathrm{~K})\left(\mathrm{P}_{\mathrm{t}}^{\prime}-\mathrm{P}_{\mathrm{S}}^{\prime}\right) /\left(\overline{\mathrm{P}_{\mathrm{t}}}-\overline{\mathrm{P}_{\mathrm{S}}}\right)
$$

were entirely representative of the hot-film signal. $\mathrm{K}$ is an empirically determined parameter; Jenkins reports typical values of 1.114 to 1.127 . In other words, Jenkins considered static pressure an instantaneous flow property.

The relationship between the shape of a pitot probe tip and its time-averaged response in a turbulent flow was studied by Becker and Brown (ref. 5). Different probe geometries (tapered, round and square nosed) were found to have markedly different response to turbulence. This behavior may be used to advantage; an estimate of rootmean-square turbulence may be made by comparing the 
steady state stagnation pressures recorded by probes of differing geometry.

Walshe and Garner (ref. 6) studied the behavior of different probe configurations such as Pitot, Kiel, and five-hole probes in turbulent flow. They concluded that five-hole probes respond similarly to cowled pitot probes when measuring highly turbulent flow. Under these circumstances the mean dynamic pressure was judged to have up to $10 \%$ error. Flow angularity correlations were made comparing the five-hole probe used in a nulling and non-nulling configuration with the angularity of peak pressures obtained with an axial probe. Discrepancies were found between the three procedures when applied to turbulent flow.

Whitmore (refs. 7 and 8) has extended the concept of multi-hole pressure probes to the construction of flushmounted airdata systems. The HI-FADS system utilizes 25 flush pressure ports mounted on an aircraft nosecone. It was calibrated for flight at both high angle of attack and large sideslip angles. At a $25 \mathrm{~Hz}$ computation rate, output from the HI-FADS system correlated well with flight data. One must note, however, that the flow over the aircraft nosecone is laminar.

In summary, the existing literature implies the following: (1) when measuring a flow with frequency content beneath the pneumatic attenuation limit of the probe, quasi-steady techniques can measure both mean and unsteady flow properties; (2) above this frequency limit, the probes tend to overestimate the mean dynamic pressure; and (3) uncertainty exists when mean flow angularity measurements are made in turbulent flow.

Despite these limitations, a probe calibrated under steady flow conditions remains a useful tool for measurement. Given sufficient frequency response, it can provide a metric of flow unsteadiness. A method of probe computations consistent with steady state theory will be developed below.

\section{Potential Flow Model}

Whitmore (ref. 8) has demonstrated the applicability of a simple, hemispherical potential flow model to derive expressions for determining flow angularity. The pressure coefficient at the surface of a hemisphere is:

$$
C_{P}(\Theta)=5 / 4+9 / 4 \cos ^{2}(\Theta)
$$

where $\Theta$ is the total flow incidence angle at the surface. Following Whitmore's derivation, the pressures on quasihemispherical shapes, such as the cone probe or an aircraft nose cone, may be approximated by:

$$
C_{P}(\Theta)=\varepsilon+\gamma \cos ^{2}(\Theta)
$$

where $\varepsilon$ and $\gamma$ are empirically determined for a given probe shape.

The port pressures are:

$$
P_{i}=P_{S}+q\left[\cos ^{2}\left(\Theta_{i}\right)+\varepsilon \sin ^{2}\left(\Theta_{i}\right)\right]
$$

where

$$
\begin{aligned}
\Theta_{i}= & \cos \alpha \cos \beta \cos \lambda_{i}+\sin \beta \sin \lambda_{i} \sin \phi_{i} \\
& +\sin \alpha \cos \beta \sin \lambda_{i} \cos \phi_{i}
\end{aligned}
$$

and $\lambda_{i}$ and $\phi_{i}$ are coordinates of the pressure ports on the probe tip.

Figures 2(a) and 2(b) show the response of three meridional probe tip pressure ports (ports 1,7 , and 4 ) as a function of pitch angle, $\alpha$. In figure 2(a), the pressures are computed using equation 3 where the empirical coefficient, $\varepsilon$, is chosen so that the computed pressures at $\alpha=0$ are consistent with experiment. Figure 2(b) shows the actual probe pressures measured using a $45^{\circ}$ cone probe. A comparison reveals that the empirical potential flow model provides qualitative, but not quantitative, prediction of the port pressures.

\section{Method of Triples}

The potential flow model may be used to further examine the possibility of contriving dimensionless pressure ratios which are solely functions of flow angularity. The simplest pressure ratios, or "triples," involve the pressures at three distinct ports:

$$
\Pi_{i j k}=\left(P_{i}-P_{j}\right) /\left(P_{j}-P_{k}\right) \quad i \neq j \neq k
$$

If one assumes that the pressures are governed by the potential flow model, equation 3, the pressures are:

$$
\Pi_{\mathrm{ijk}}=\left(\cos ^{2} \Theta_{\mathrm{i}}-\cos ^{2} \Theta_{\mathrm{j}}\right) /\left(\cos ^{2} \Theta_{\mathrm{j}}-\cos ^{2} \Theta_{\mathrm{k}}\right)
$$

Figures 3(a) and 3(b) demonstrate the differences between the actual probe response and simplified model given a pressure triple based upon three meridional pressure ports (ports 1,7, and 4). Both the experimental data and the analytical model exhibit qualitative similarities including a singularity at a flow angularity of approximately onehalf the probe cone angle.

The non-meridional triples are significantly less well behaved. As can be seen in figures 4(a) and 4(b), some of the non-meridional triples are multiply valued functions.

$\Pi_{123}=0.25$ may correspond to a flow angularity of $-30^{\circ}$, $-20^{\circ},-7^{\circ}$, and, possibly, $+2^{\circ}$. 
To reduce the system from an overdetermined state to one of one-to-one mapping, piecewise continuous pressure ratio functions must be used. Gallington (ref. 9) has found a complex, dimensionless pressure ratio is well behaved over a wide region of calibration space. A pair of pressure ratios, sensitive to pitch and yaw, are defined as functions of all seven pressures:

$$
\begin{aligned}
& \mathrm{C}_{\mathrm{P} \alpha 7}=\mathrm{C}_{\mathrm{Pta}}+\left(\mathrm{C}_{\mathrm{Ptb}}-\mathrm{C}_{\mathrm{Ptc}}\right) / 2 \\
& \mathrm{C}_{\mathrm{P} \beta 7}=(1 / \sqrt{3})\left(\mathrm{C}_{\mathrm{Ptb}}+\mathrm{C}_{\mathrm{Ptc}}\right)
\end{aligned}
$$

where

$$
\begin{aligned}
\mathrm{C}_{\mathrm{Pta}} & =\left(\mathrm{P}_{4}-\mathrm{P}_{1}\right) /\left(\mathrm{P}_{7}-\overline{\mathrm{P}}\right) \\
\mathrm{C}_{\mathrm{Ptb}} & =\left(\mathrm{P}_{3}-\mathrm{P}_{6}\right) /\left(\mathrm{P}_{7}-\overline{\mathrm{P}}\right) \\
\mathrm{C}_{\mathrm{Ptc}} & =\left(\mathrm{P}_{2}-\mathrm{P}_{5}\right) /\left(\mathrm{P}_{7}-\overline{\mathrm{P}}\right) \\
\overline{\mathrm{P}} & =(1 / 6)\left(\mathrm{P}_{1}+\mathrm{P}_{2}+\mathrm{P}_{3}+\mathrm{P}_{4}+\mathrm{P}_{5}+\mathrm{P}_{6}\right)
\end{aligned}
$$

The response of these coefficients to flow angularity is shown in figures 5 and 6 . These figures show that Gallington's functions are well behaved and, more importantly, single valued over their entire calibration range. Provided that the flow over the entire probe tip is attached, all seven pressures are relevant. These coefficients directly define the flow angularity.

These calibration functions may be numerically inverted to define flow angularity, $\alpha$ and $\beta$, in terms of the two coefficients. A pair of transfer functions, $f_{\alpha}$ and $f_{\beta}$, may be computed:

$$
\begin{gathered}
\alpha=f_{\alpha}\left(C_{P \alpha i}, C_{P \beta i}\right) \\
\beta=f_{\beta}\left(C_{P \alpha i}, C_{P \beta i}\right)
\end{gathered}
$$

They are tabulated in figures 7 (a) and 7(b).

The inversion process was accomplished using the following procedure. First, a subroutine was written to interpolate values of $C_{P \alpha j}$ and $C_{P \beta i}$ from the calibration data set given any arbitrary values of $\alpha$ and $\beta$. This subroutine was embedded in a minimization algorithm designed to find an $\alpha$ and $\beta$ such that the interpolated values of $C_{P \alpha i}, C_{P \beta i}$ match "target" values of these functions. In other words, to find the $\alpha$ and $\beta$ corresponding to a given pair of values of the calibration functions:
Step 1: Choose a target value for the flow angularity coefficients $\mathrm{C}_{\mathrm{P} \alpha \mathrm{iT}}$ and $\mathrm{C}_{\mathrm{P} \beta \mathrm{iT}}$.

Step 2: $\quad$ Minimize: $f(\alpha, \beta)$

where

$$
\begin{aligned}
f(\alpha, \beta)= & \left(C_{P \alpha i}(\alpha, \beta)-C_{P \alpha i T}\right)^{2} \\
& +\left(C_{P \beta i}(\alpha, \beta)-C_{P \beta i T}\right)^{2}
\end{aligned}
$$

Subject to: $\alpha, \beta$ bounded.

Step 3: At local minimum?

If YES, use derived $\alpha, \beta$.

If $N O$, let $\alpha, \beta$ go out of bounds $\left(\alpha=-999^{\circ}, \beta=-999^{\circ}\right)$.

Step 4: $\quad$ Insert values of $\alpha, \beta$ into the $f_{\alpha i}\left(C_{P \alpha i}, C_{P \beta i}\right)$ and $f_{\beta i}\left(C_{P \alpha i}, C_{P \beta i}\right)$ transfer function matrices.

Step 5: $\quad$ Choose new values of $\mathrm{C}_{\mathrm{P} \alpha \mathrm{iT}}$ and $\mathrm{C}_{P \beta \mathrm{i}}$ and $\mathrm{i}$.

\section{Step 6: $\quad$ Go to Step 2}

\section{High Flow Angularity Coefficients}

For the high flow angularity situation, where the flow over the probe tip may have separated, unique pressure ratios must be formulated to avoid inclusion of ports on the lee side of the probe. Six pairs of coefficients may be formulated; each excludes specific neighboring ports.

Because of the restricted domain of these functions, they need not be well defined about $\alpha=\beta=0$.

The rule set divides the calibration set into specific sectors. In Zilliac (ref. 10), the choice of sector is determined from an a priori inspection of the dominant pressure. If $P_{7}>P_{i}(i=1 \ldots 6)$, then $C_{P \alpha 7}$ and $C_{P \beta 7}$ are utilized; if $\mathrm{P}_{7}$ is not the dominant pressure, then the algorithm uses a separated flow pressure coefficient. $P_{i}$ tends to exceed $\mathrm{P}_{7}$ when the flow angularity reaches half of the cone angle $\left(22.5^{\circ}\right.$ for a $45^{\circ}$ cone probe). In reality, the flow will separate at much higher angles. This author concludes that Zilliac may have used separated flow coefficients prematurely. 
A more lenient flow separation criterion may be developed. $\mathrm{C}_{\mathrm{P \alpha} 7}$ and $\mathrm{C}_{\mathrm{P} \beta 7}$ are extremely well behaved over the entire calibration range. Consequently, even at high flow angularity $C_{P \alpha}$ and $C_{P \beta}$ are a single valued function of flow angularity. The onset of flow separation may be identified by the angle found using $\mathrm{C}_{\mathrm{Pa}} 7$ and $\mathrm{C}_{\mathrm{P} \beta 7}$.

For the case of a $45^{\circ}$ cone probe, a cut-off point of $30^{\circ}$ included angle was used.

Gallington (ref. 9) also developed a set of secondary flow angularity coefficients which have the property of being bounded, $\left|C_{P \alpha i}\right|<2$ and $\left|C_{P \beta i}\right|<2$, and free from singularities over their respective useful calibration ranges.

Sector 1 ( $P_{1}$ dominant $)$

$$
\begin{aligned}
& C_{P \alpha 1}=\Pi_{1267}=\left(P_{1}-P_{7}\right) /\left[P_{1}-\left(P_{2}+P_{6}\right) / 2\right] \\
& C_{P \beta 1}=\Pi_{126}=\left(P_{6}-P_{2}\right) /\left[P_{1}-\left(P_{2}+P_{6}\right) / 2\right]
\end{aligned}
$$

Sector 2 ( $\mathrm{P}_{2}$ dominant)

$$
\begin{aligned}
& C_{P \alpha 2}=\Pi_{1237}=\left(P_{2}-P_{7}\right) /\left[P_{2}-\left(P_{1}+P_{3}\right) / 2\right] \\
& C_{P \beta 2}=\Pi_{123}=\left(P_{1}-P_{3}\right) /\left[P_{2}-\left(P_{1}+P_{3}\right) / 2\right]
\end{aligned}
$$

Sector 3 ( $\mathrm{P}_{3}$ dominant)

$$
\begin{aligned}
& C_{P \alpha 3}=\Pi_{2347}=\left(P_{3}-P_{7}\right) /\left[P_{3}-\left(P_{2}+P_{4}\right) / 2\right] \\
& C_{P \beta 3}=\Pi_{234}=\left(P_{2}-P_{4}\right) /\left[P_{3}-\left(P_{2}+P_{4}\right) / 2\right]
\end{aligned}
$$

\section{Sector $4\left(\mathrm{P}_{4}\right.$ dominant $)$}

$$
\begin{aligned}
& C_{P \alpha 4}=\Pi_{1237}=\left(P_{4}-P_{7}\right) /\left[P_{2}-\left(P_{1}+P_{3}\right) / 2\right] \\
& C_{P \beta 4}=\Pi_{345}=\left(P 3-P_{5}\right) /\left[P_{4}-\left(P_{3}+P_{5}\right) / 2\right]
\end{aligned}
$$

Sector 5 ( $\mathrm{P}_{5}$ dominant)

$$
\begin{aligned}
& C_{P \alpha 5}=\Pi_{4567}=\left(P_{5}-P_{7}\right) /\left[P_{5}-\left(P_{4}+P_{6}\right) / 2\right] \\
& C_{P \beta 5}=\Pi_{456}=\left(P_{4}-P_{6}\right) /\left[P_{5}-\left(P_{4}+P_{6}\right) / 2\right]
\end{aligned}
$$

Sector 6 ( $\mathrm{P}_{6}$ dominant)

$$
\begin{aligned}
& C_{P \alpha 6}=\Pi_{1567}=\left(P_{6}-P_{7}\right) /\left[P_{6}-\left(P_{5}+P_{1}\right) / 2\right] \\
& C_{P \beta 6}=\Pi_{156}=\left(P_{5}-P_{1}\right) /\left[P_{6}-\left(P_{5}+P_{1}\right) / 2\right]
\end{aligned}
$$

Figures 8(a) and 8(b) demonstrate the behavior of $C_{P \alpha 1}$ for large positive $\alpha$, and $\mathrm{C}_{\mathrm{P} \alpha 4}$ for large negative $\alpha$. Each of these functions is singular about $\alpha=0$ and shows strong asymptotic behavior for $\alpha>30^{\circ}$.

\section{Algorithm-Part I: Flow Angularity}

The processing algorithm may be implemented as follows:

Step 1: Compute the basic flow angularity coefficients: $\mathrm{C}_{P \alpha 7}$ and $\mathrm{C}_{P \beta}$.

Step 2: If they are outside the range of the sector 7 transfer function table, go to Step 5.

Step 3: Perform bilinear interpolation on the sector 7 tables to determine $\alpha$ and $\beta$.

Step 4: $\quad$ Are $\alpha$ and $\beta$ indicative of separated flow over the probe tip?

If YES, continue on to Step 5.

If $\mathrm{NO}$, then we are done.

Step 5: Determine which pressure port has the largest positive pressure (the "dominant hole")-this defines the sector, $i$.

Step 6: Compute the appropriate coefficients: $\mathrm{C}_{\mathrm{P} \alpha \mathrm{i}}, \mathrm{C}_{\mathrm{PBi}}$.

Step 7: Are these coefficients within the range of the tables? If not, then we have BAD data.

Step 8: $\quad$ Perform bilinear interpolation on the separated flow tables to determine $\alpha$ and $\beta$.

\section{Computation of Static and Total Pressure}

With the flow angularity determined, the static and total pressure may be inferred from ratio of peak pressure to the mean of the pressures governed by unseparated flow. Static and total pressure coefficients are formulated in terms of the actual tunnel static pressure, $P_{S}$, and total pressure, $P_{t}$ :

$$
\begin{aligned}
& \mathrm{C}_{\mathrm{Ps}_{1}}=\left[\left(\mathrm{P}_{2}+\mathrm{P}_{6}\right) / 2-\mathrm{P}_{\mathrm{s}}\right] /\left[\mathrm{P}_{1}-\left(\mathrm{P}_{2}+\mathrm{P}_{6}\right) / 2\right] \\
& \mathrm{C}_{\mathrm{Pt} 1}=\left(\mathrm{P}_{1}-\mathrm{P}_{t}\right) /\left[\mathrm{P}_{1}-\left(\mathrm{P}_{2}+\mathrm{P}_{6}\right) / 2\right] \\
& \mathrm{C}_{\mathrm{Ps} 2}=\left[\left(\mathrm{P}_{1}+\mathrm{P}_{3}\right) / 2-\mathrm{P}_{\mathrm{s}}\right] /\left[\mathrm{P}_{2}-\left(\mathrm{P}_{1}+\mathrm{P}_{3}\right) / 2\right] \\
& \mathrm{C}_{\mathrm{Pt} 2}=\left(\mathrm{P}_{2}-\mathrm{P}_{\mathrm{t}}\right) /\left[\mathrm{P}_{2}-\left(\mathrm{P}_{1}+\mathrm{P}_{3}\right) / 2\right] \\
& \mathrm{C}_{\mathrm{Ps} 3}=\left[\left(\mathrm{P}_{2}+\mathrm{P}_{4}\right) / 2-\mathrm{P}_{\mathrm{s}}\right] /\left[\mathrm{P}_{3}-\left(\mathrm{P}_{2}+\mathrm{P}_{4}\right) / 2\right] \\
& \mathrm{C}_{\mathrm{Pt} 3}=\left(\mathrm{P}_{3}-\mathrm{P}_{t}\right) /\left[\mathrm{P}_{3}-\left(\mathrm{P}_{2}+\mathrm{P}_{4}\right) / 2\right] \\
& \mathrm{C}_{\mathrm{Ps} 4}=\left[\left(\mathrm{P}_{3}+\mathrm{P}_{5}\right) / 2-\mathrm{P}_{\mathrm{s}}\right] /\left[\mathrm{P}_{4}-\left(\mathrm{P}_{3}+\mathrm{P}_{5}\right) / 2\right] \\
& \mathrm{C}_{\mathrm{Pt} 4}=\left(\mathrm{P}_{4}-\mathrm{P}_{\mathrm{t}}\right) /\left[\mathrm{P}_{4}-\left(\mathrm{P}_{3}+\mathrm{P}_{5}\right) / 2\right] \\
& \mathrm{C}_{\mathrm{Ps}_{5}}=\left[\left(\mathrm{P}_{4}+\mathrm{P}_{6}\right) / 2-\mathrm{P}_{\mathrm{s}}\right] /\left[\mathrm{P}_{5}-\left(\mathrm{P}_{4}+\mathrm{P}_{6}\right) / 2\right] \\
& \mathrm{C}_{\mathrm{Pt} 5}=\left(\mathrm{P}_{5}-\mathrm{P}_{\mathrm{t}}\right) /\left[\mathrm{P}_{5}-\left(\mathrm{P}_{4}+\mathrm{P}_{6}\right) / 2\right]
\end{aligned}
$$




$$
\begin{aligned}
& \mathrm{C}_{\mathrm{Ps} 6}=\left[\left(\mathrm{P}_{5}+\mathrm{P}_{1}\right) / 2-\mathrm{P}_{\mathrm{s}}\right] /\left[\mathrm{P}_{6}-\left(\mathrm{P}_{5}+\mathrm{P}_{1}\right) / 2\right] \\
& \mathrm{C}_{\mathrm{Pt} 6}=\left(\mathrm{P}_{6}-\mathrm{P}_{\mathrm{t}}\right) /\left[\mathrm{P}_{6}-\left(\mathrm{P}_{5}+\mathrm{P}_{1}\right) / 2\right]
\end{aligned}
$$

and

$$
\begin{aligned}
& C_{P_{s} 7}=\left(\bar{P}-P_{s}\right) /\left(P_{7}-\bar{P}\right) \\
& C_{P_{t} 7}=\left(\bar{P}-P_{t}\right) /\left(P_{7}-\bar{P}\right)
\end{aligned}
$$

In theory, the static and total pressure can be reconstructed for any flow angularity. For example, in unseparated flow the static and total pressures are reconstructed from the probe pressures and estimated flow angularity as:

$$
P_{t}=P_{7}-C_{P t}(\alpha, \beta)\left(P_{7}-\bar{P}\right)
$$

and

$$
P_{S}=P_{7}-C_{P s} 7(\alpha, \beta)\left(P_{7}-\bar{P}\right)
$$

The reconstruction coefficients are well defined over their useful ranges; select matrices are tabulated in figures $9(a)-9(d)$.

Special care must be taken when recording the data used for calibration to obtain a correct estimate of static pressure. While total pressure remains invariant in an inviscid flow, an actual wind tunnel will exhibit an axial static pressure gradient. If an incorrect estimate of static pressure is made at calibration time, the probes will estimate an incorrect dynamic pressure in operation.

\section{Algorithm-Part II: Static and Total Pressure}

In terms of the computational algorithm, recall that the flow angularity computations have determined both the appropriate sector, $i$, and the flow angularity $(\alpha, \beta)$. These coefficients are used to perform a pair of bilinear interpolations upon the appropriate static and total pressure coefficient matrix.

Step 9: $\quad$ Interpolate $C_{P s i}$ from $\alpha, \beta$ and $i$.

Step 10: Compute static pressure, $P_{S}$.

Step 11: Interpolate $C_{P T i}$ from $\alpha, \beta$ and $i$.

Step 12: Compute total pressure, $P_{t}$.

Implementation of this algorithm is extremely efficient. On a 486 PC, a computational throughput of several hundred reductions per second is realized. A real-time computation of flow properties is possible.

\section{Experimental Verification Turbulent Wake Flow}

Results from two recent experiments will be shown. Both are studies of the downstream wakes of circular cylinders. Figures 10 (a) and 10 (b) derive from an experiment made in a small developmental wind tunnel at the Ames Fluid Mechanics Laboratory; figures 11 and 12 derive from an experiment made at the Ames 7 - by 10-Foot Subsonic Wind Tunnel No. 1. The natural pneumatic dissipation of the probe tubing limits the frequency response to approximately $2 \mathrm{~Hz}$. The Karman vortex street shed by the cylinders occurs at a much greater frequency: approximately $250 \mathrm{~Hz}$ for the $\mathrm{Re}=40,000$ experiment and approximately $60 \mathrm{~Hz}$ for the $\mathrm{Re}=400,000$ experiment. The probe will consider the vortex street a source of unsteady flow.

Figure 10(a) has the probe located 1.5 diameters downstream of the cylinder. The two traces correspond to the total pressure in the free stream and the total pressure with the probe immersed to the side of the near-field vortex street. The probe is sampled first at $200 \mathrm{~Hz}$, then at $100 \mathrm{~Hz}$; both in excess of the probe's low-pass pneumatic limit. It can be seen that the probe gives a uniform response to either a steady state or turbulent flow.

Figure $10(\mathrm{~b})$ is of similar geometry, but with the probe moved farther back, to $x / d=5.5$. The integration time is extended to 2.5 seconds, for an effective sample rate of $0.4 \mathrm{~Hz}$. The values for total pressure remain constant. These two figures imply that the probe exhibits essentially steady state behavior when immersed in an unsteady flow with a frequency content far above the probe limit.

Additional data were taken to compare the performance of the seven-hole probe against a hot-film anemometer. For this experiment, the seven-hole probe was configured as it is to make two-dimensional wake surveys. The probe is slowly ( 0.5 inch per second) translated across the wake. Flow properties are measured in real time (three samples of 0.25 second integration time per second), and then gated to the desired spatial resolution.

Figure 11 shows that there is close agreement between the seven-hole probe and hot-film anemometer in the freestream velocity measurements. However, the seven-hole probe tends to consistently overestimate the velocity when immersed in the vortex street. There is some scatter in the seven-hole data, attributable to the short-time integration interacting with buffeting of the probe boom (unavoidable in production testing). Figure 12 reveals that the root-mean-square unsteady velocity levels in the vortex street are as high as $25 \%$ of the local mean 
velocity. The overprediction of dynamic pressure, however unwelcome, is consistent with Goldstein (ref. 3), Becker (ref. 5), and Walshe (ref. 6).

\section{Error Analysis}

A Monte Carlo simulation may be made to assess the sensitivity of the seven-hole probe algorithm to random error. A series of perturbations of increasing magnitude were statistically applied to basic pressure patterns corresponding to a flow angularity of $\alpha=0^{\circ}, \beta=0^{\circ}$ and $\alpha=20^{\circ}, \beta=-20^{\circ}$, respectively. The results are shown in table 1 . A $1 \%$ deviation in port pressures tends to lead to an uncertainty in flow angularity of approximately $2^{\circ}$ at low flow angles, but tends toward unpredictably large excursions at high flow angularity; this is due to the shallow slopes of the separated-flow flow-angularity functions. Consequently, it is desirable to restrict the probe to as narrow a range of flow angularity as possible.

\section{Concluding Remarks}

Seven-hole cone probes are an accepted means to measure the essential mean properties of fluid flow. A rapid computational algorithm which incorporates as few as four bilinear interpolations and no more than six interpolations ( 16 to 24 array references and simple arithmetic) is presented. This has produced a computational algorithm efficient enough to allow the flow properties to be implemented in real time. Unsteady flow properties with frequency content below the pneumatic limit of the probe may be resolved. Higher frequency unsteady flow will tend to bias the probe into indicating a dynamic pressure in excess of the actual value. Over the region of calibration space where the flow has not separated, the flow angularity computations are robust. At high flow angularity, the separated flow coefficients become very sensitive to small perturbations. The computed flow directionality at high flow angularity is best left for qualitative, rather than quantitative, presentation.

Some issues require further investigation. There is a need to:

- Develop design guidelines to define the optimal balance between probe size and frequency response.

- Determine the limiting frequency where a quasisteady calibration can be applied.

- Find well behaved flow angularity coefficients which are more tolerant of random error, at high flow angularity, than the Gallington set. (This author has tried many with little success.)
- Use seven-hole probes, in the absence of better flow angularity coefficients, in a semi-nulling configuration (where the peak flow angles are restricted).

- Address the meaning of mean flow properties, particularly static pressure, in a turbulent flow.

- Address the concept of unsteady, bandwidth limited static pressure, as reported by a real time multi-hole pitot probe, in turbulent flows.

\section{References}

1. Prandtl, L.; and Tietjens, O. G.: Applied Hydro- and Aeromechanics. McGraw-Hill, New York, 1934.

2. Schulze, W. M.; Ashby, G. C.; and Erwin, J. R.: Several Combination Probes for Surveying Static and Total Pressure and Flow Direction. NACA TN-2830, 1952.

3. Goldstein, S.: A Note on the Measurement of Total Head and Static Pressure in a Turbulent Stream. Proc. Roy. Soc. London A, vol. 155, 1936, pp. 570-575.

4. Jenkins, R. C.: Effects of Pitot Probe Shape on Measurement of Flow Turbulence. AIAA J., vol. 25, no. 6, 1987, pp. 889-892.

5. Becker, H. A.; and Brown, A. P. G.: Response of Pitot Probes in Turbulent Streams. J. Fluid Mech., vol 62, pt. 1, 1974, pp. 85-114.

6. Walsh, D. E.; and Garner, H. C.: Usefulness of Various Pressure Probes in Fluctuating LowSpeed Flow. British ARC Rpt. 21714, FM 2917, Feb. 1960.

7. Whitmore, S. A.; Moes, T. R.; and Larson, T. J.: Preliminary Results from a Subsonic High Angle-of-Attack Flush Airdata Sensing (HI-FADS) System: Design, Calibration and Flight-Test Evaluation. NASA TM-101713, 1990.

8. Whitmore, S. A.; and Moes, T. R.: Failure Detection and Fault Management Techniques for a Pneumatic High-Angle of Attack Flush Airdata Sensing System. NASA TM-4335, 1992.

9. Gallington, R. W.: Measurement of Very Large Flow Angles with Non-Nulling Seven-Hole Probes. USAFA-TR-80-17, 1980.

10. Zilliac, G. G.: Calibration of Seven Hole Pressure Probes for Use in Fluid Flows with Large Angularity. NASA TM-102200, 1990. 
Table 1. Monte Carlo error analysis-seven-hole probe computational algorithm

\begin{tabular}{ccc}
\hline $\begin{array}{c}\text { Probe pressure } \\
\text { port perturbation }\end{array}$ & $\begin{array}{c}\alpha=0^{\circ} / \beta=0^{\circ} \\
\text { RMS error }\end{array}$ & $\begin{array}{c}\alpha=20^{\circ} / \beta=-20^{\circ} \\
\text { RMS error }\end{array}$ \\
\hline $\pm 0 \%$ & $\pm 0^{\circ}$ & $\pm 0^{\circ}$ \\
$\pm 1 / 4 \%$ & $\pm 0.5^{\circ} \pm 0.5^{\circ}$ & $\pm 1.0^{\circ} \pm 0.7^{\circ}$ \\
$\pm 1 / 2 \%$ & $\pm 1^{\circ} \pm 1^{\circ}$ & $\pm 2^{\circ} \pm 1.5^{\circ}$ \\
$\pm 1 \%$ & $\pm 2^{\circ} \pm 2^{\circ}$ & $\pm 7.5^{\circ} \pm 16^{\circ}$ \\
\hline
\end{tabular}

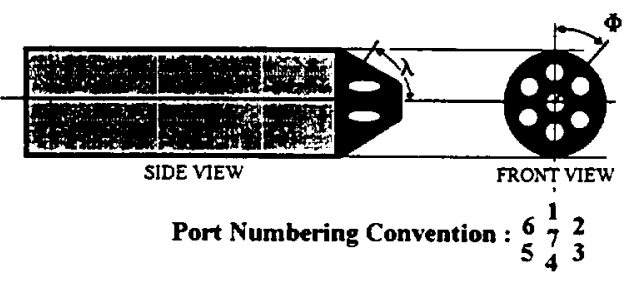

Figure 1. General schematic of a seven-hole probe.

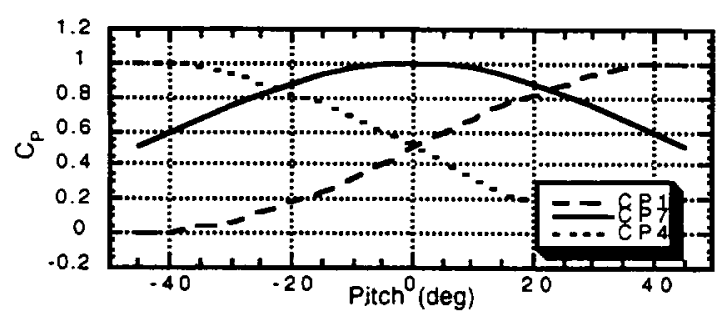

Figure 2(a). Pressure due to pitch. Empirical (potential flow) model. Meridional pressure ports (1, 7, and 4). $\varepsilon=0$; chosen to match experimental data at $0^{\circ}$ pitch angle.

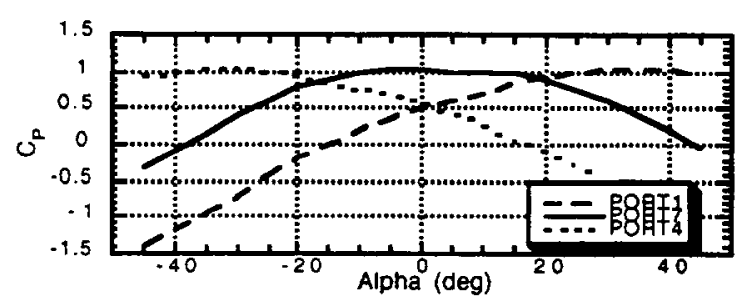

Figure 2(b). Pressure due to pitch. Experimental results. Meridional pressure ports $(1,7$, and 4$))$. Pressures from $45^{\circ}$ seven-hole cone probe calibration.

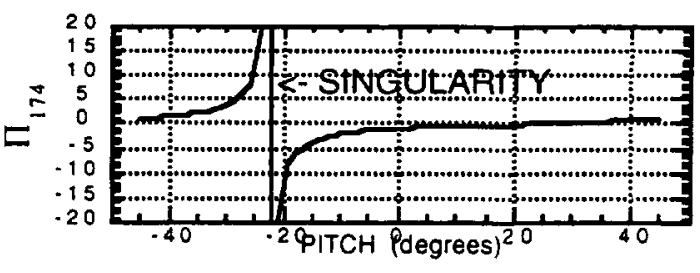

Figure 3(a). Simple, dimensionless pitch-sensitive pressure ratio as a function of pitch. Meridional "triple" $\left.\left(\Pi_{174}=\left(P_{1}-P_{7}\right)\right)\left(P_{7}-P_{4}\right)\right)$. Empirical (potential flow) model.

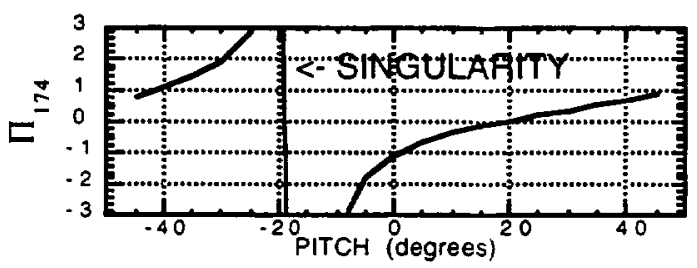

Figure 3(b). Simple, dimensionless pitch-sensitive pressure ratio as a function of pitch. Meridional "triple" $\left.\left(\Pi_{174}=\left(P_{1}-P_{7}\right)\right)\left(P_{T}-P_{4}\right)\right)$. Experimental results from $45^{\circ}$ cone probe.

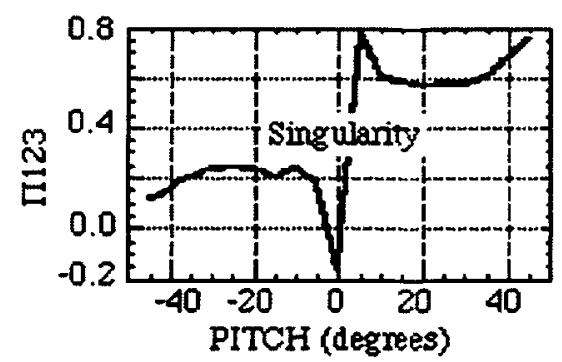

Figure 4(a). Simple, dimensionless pitch-sensitive pressure ratio as a function of pitch. Non-meridional "triple" $\left(\Pi_{123}\right)$. Experimental data from $45^{\circ}$ cone probe. Note singularity near $0^{\circ}$ pitch angle.

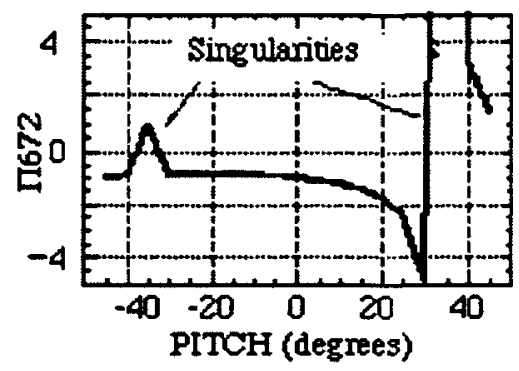

Figure 4(b). Simple, dimensionless pitch-sensitive pressure ratio as a function of pitch. Non-meridional "triple" $\left(\Pi_{672}\right)$. Experimental data from $45^{\circ}$ cone probe. Note singularities near $-35^{\circ}$ and $+35^{\circ}$ pitch angle. 


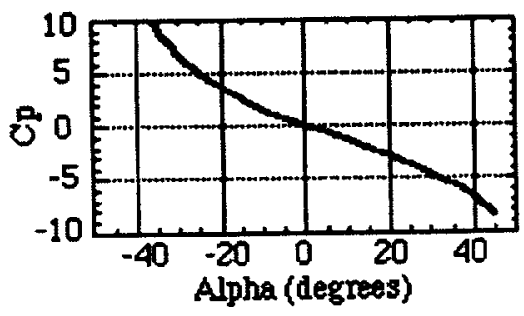

Figure 5. Complex, dimensionless pitch-sensitive pressure ratio, $C_{P \alpha}$, as a function of pitch $(\beta=0)$. Experimental results from $45^{\circ}$ cone probe.

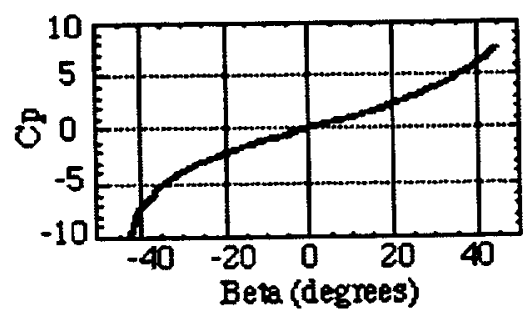

Figure 6. Complex, dimensionless yaw-sensitive pressure ratio, $C_{P \beta 7}$, as a function of yaw $(\alpha=0)$. Experimental results from $45^{\circ}$ cone probe.

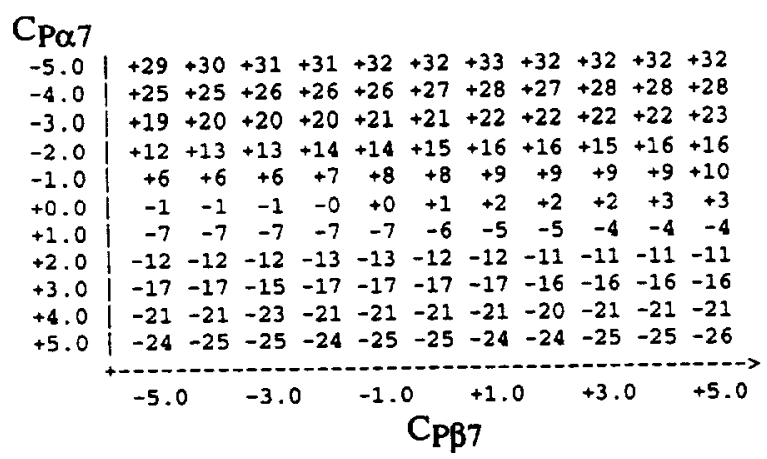

Figure $7(a)$. Pitch transfer function for unseparated flow. Sector 7. $\alpha=t_{\alpha}\left(C_{P \alpha}, C_{P \beta 7}\right)$.

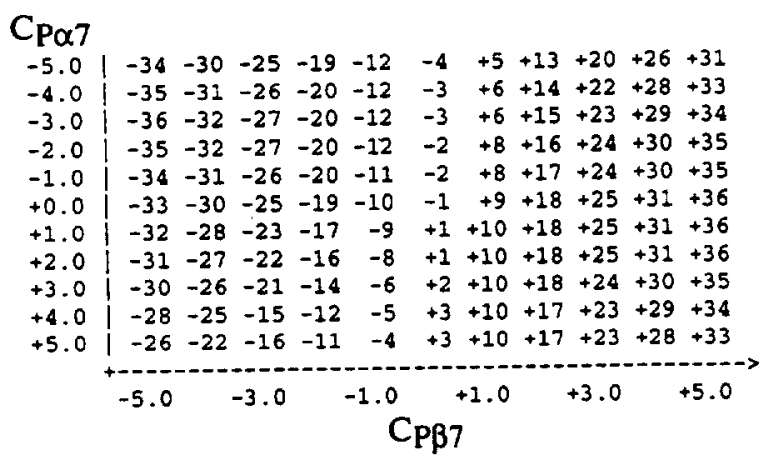

Figure $7(b)$. Yaw transfer function for unseparated flow. Sector 7. $\beta=f_{\beta}\left(C_{P \alpha}, C_{P \beta 7}\right)$.

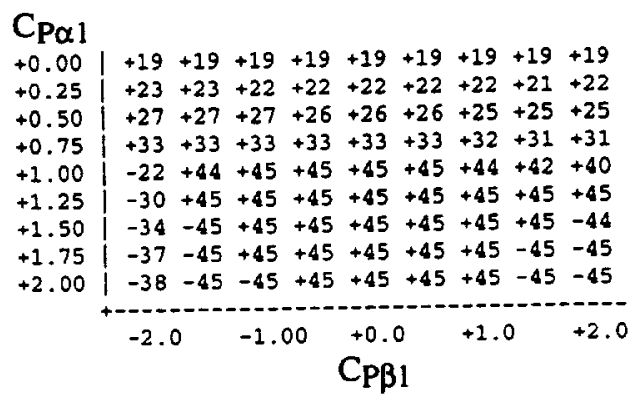

Figure $7(c)$. Pitch transfer function for separated flow. Sector 1. $\alpha=f_{\alpha}\left(C_{P \alpha 1}, C_{P \beta 1}\right)$.

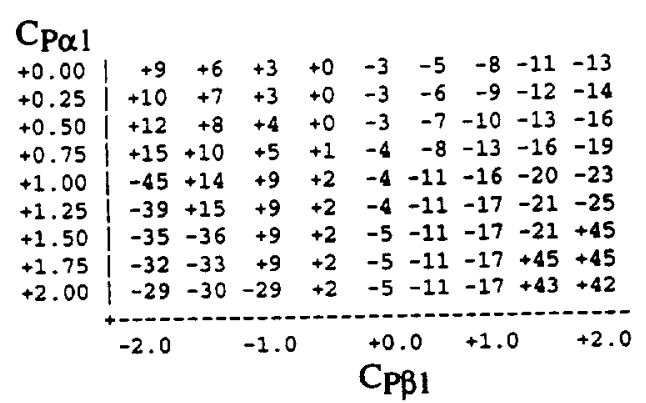

Figure $7(d)$. Yaw transfer function for separated flow. Sector 1. $\beta=f_{\beta}\left(C_{P \alpha 1}, C_{P \beta 1}\right)$.

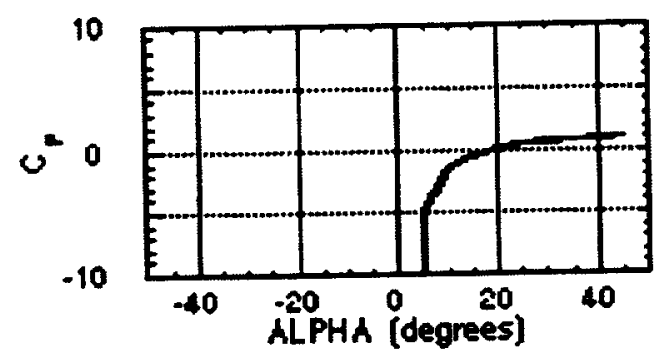

Figure 8(a). Complex, dimensionless pitch-sensitive separated flow pressure ratio, $C_{P \alpha 1}$, as a function of pitch $(\beta=0)$.

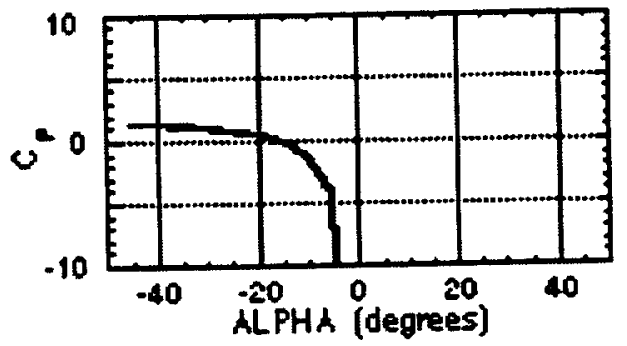

Figure 8(b). Complex, dimensionless pitch-sensitive, separated flow pressure ratio, $C_{P \alpha 4}$, as a function of pitch $(\beta=0)$. 


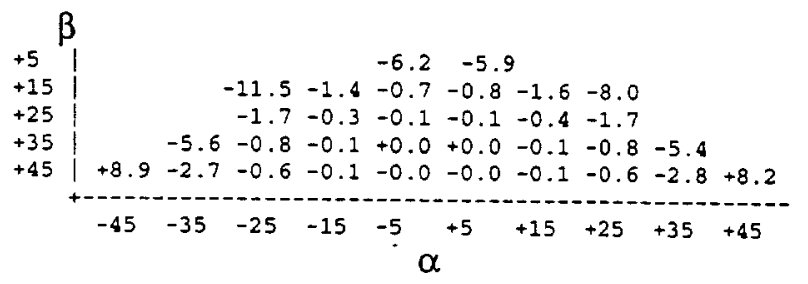

Figure 9(a). Static pressure coefficients for separated flow. Sector 1. $C_{P S 1}$.

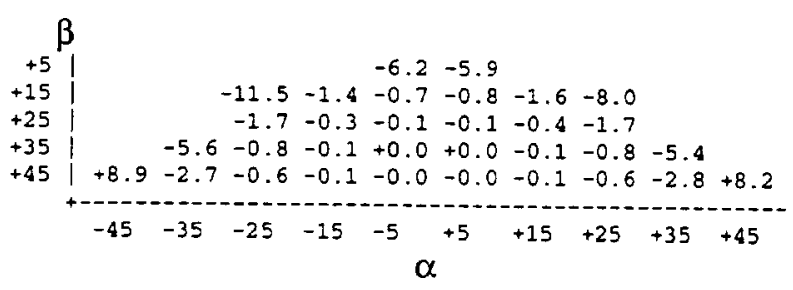

Figure 9(b). Total pressure coefficients for separated flow. Sector 1. CPt1.

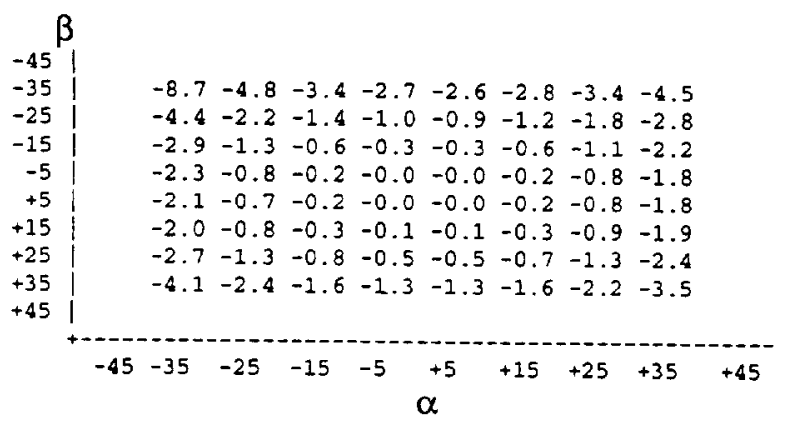

Figure 9(c). Static pressure coefficients for unseparated flow. Sector 7. CPs7.

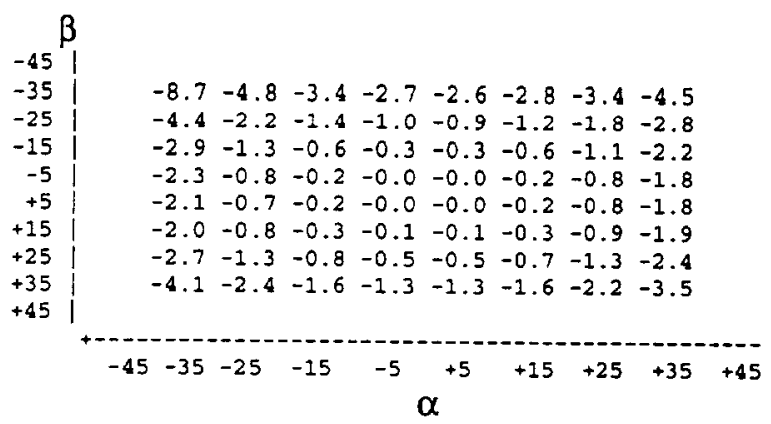

Figure $9(d)$. Total pressure coefficients for unseparated flow. Sector 7. CPt7.

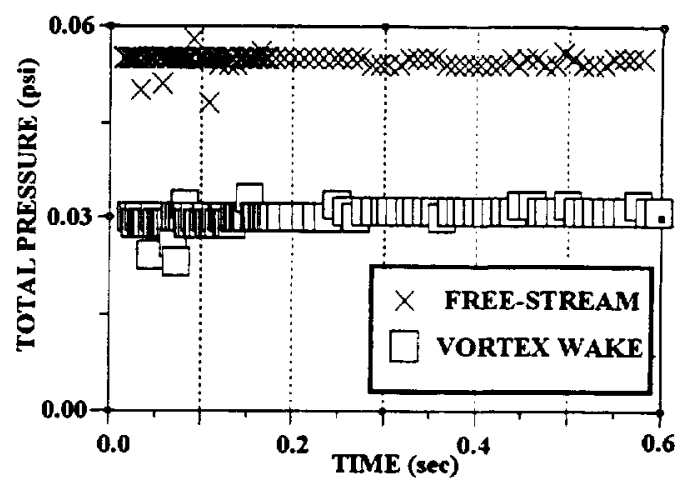

Figure 10(a). Time history of flow. Data from cylinder wake test $(R e=40,000, x / d=1.5)$. Two spatial locations chosen-one in the free stream, the other in the vortex wake. 200 and $100 \mathrm{~Hz}$ sampling rates. Note minor "scatter" for $200 \mathrm{~Hz}$ sample rate (due to electrical noise) and minimal "scatter" for $100 \mathrm{~Hz}$ sample rate. There is no appreciable detection of the flow unsteadiness in the vortex wake.

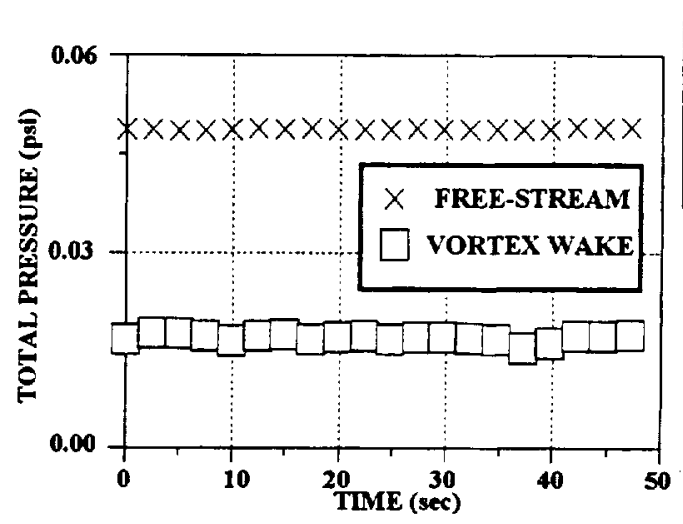

Figure 10(b). Time history in wake. Data from cylinder wake test $(R e=40,000, x / d=5.5)$. Two spatial locations chosen-one in the free stream, the other in the vortex wake. 2.5 second integration time, $0.4 \mathrm{~Hz}$ sample rate. 


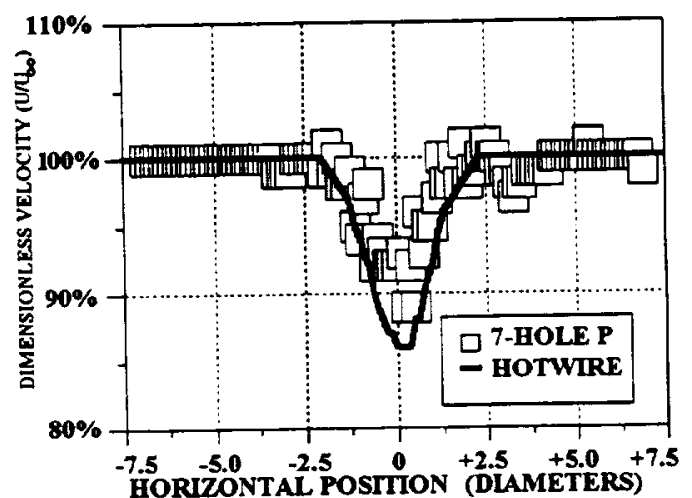

Figure 11. Axial velocity in cylinder wake $(R e=400,000$, $x^{\prime d}=7.5$ ). Seven-hole and hot-film anemometry. $4 \mathrm{~Hz}$ data acquisition rate $(0.1$ second pressure integration time).

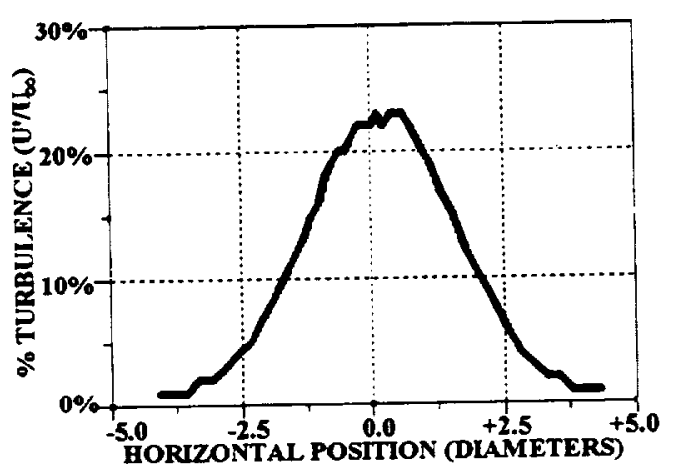

Figure 12. Turbulence in cylinder wake $(R e=400,000$, $x / d=7.5$ ) from hot-film anemometry. 


\section{Appendix A-Additional Figures}

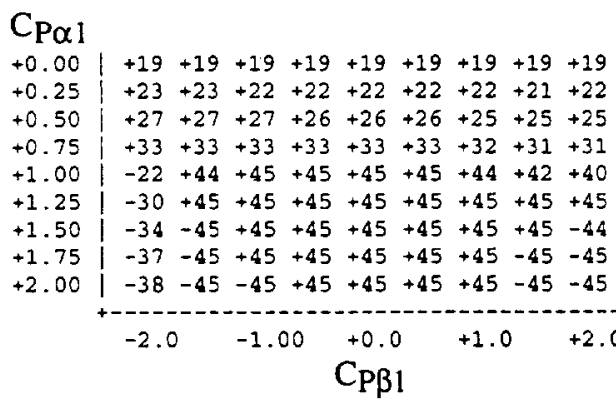

Figure 13(a). Transfer function for separated flow. $\alpha=f_{\alpha}\left(C_{P \alpha 1}, C_{P \beta 1}\right)$.

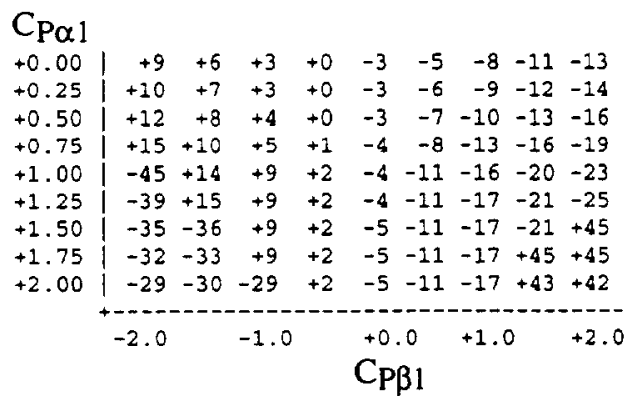

Figure 13(b). Transfer function for separated flow. $\beta=f_{\beta}\left(C_{P \alpha 1}, C_{P \beta 1}\right)$.

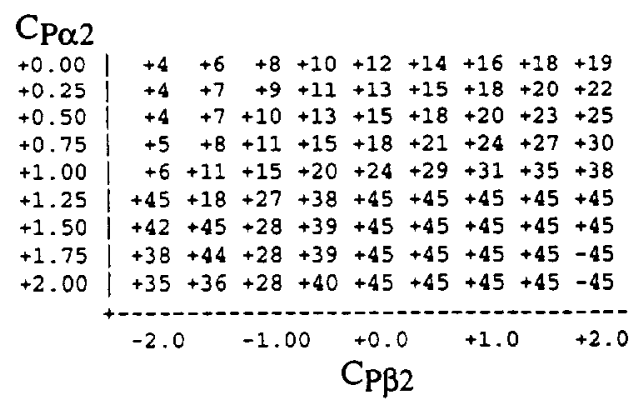

Figure 13(c). Transfer function for separated flow. $\alpha=f_{\alpha}\left(C_{P \alpha 2}, C_{P \beta 2}\right)$.

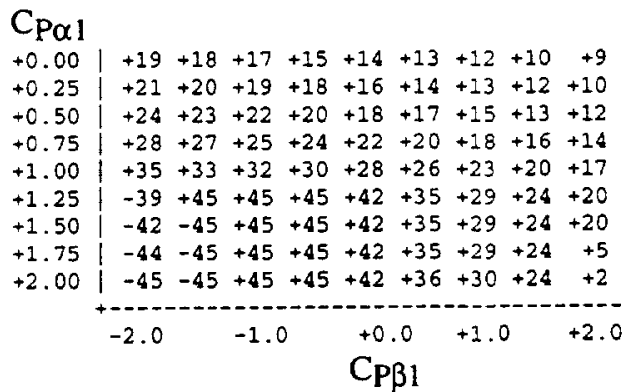

Figure 13(d). Transfer function for separated flow. $\beta=f_{\beta}\left(C_{P \alpha 2}, C_{P \beta 2}\right)$.

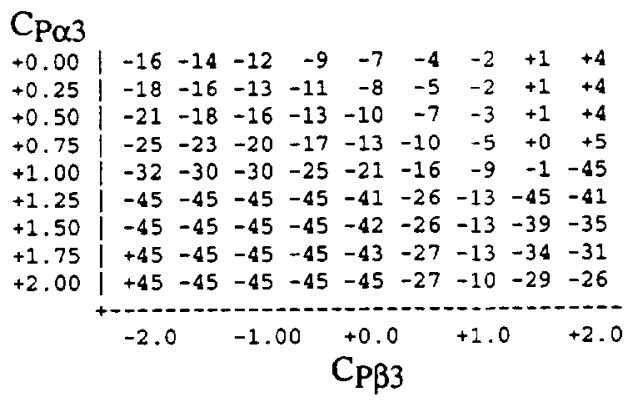

Figure 13(e). Transfer function for separated flow. $\alpha=f_{\alpha}\left(C_{P \alpha 3}, C_{P \beta 3}\right)$.

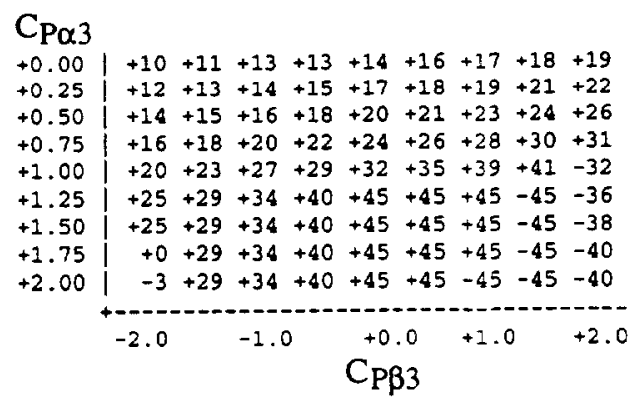

Figure 13(f). Transfer function for separated flow. $\beta=f_{\beta}\left(C_{P \alpha 2}, C_{P \beta 2}\right)$. 


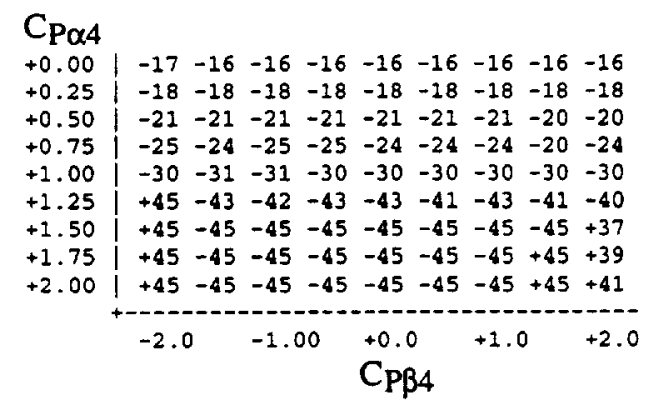

Figure $13(g)$. Transfer function for separated flow. $\alpha=f_{\alpha}\left(C_{P \alpha 4}, C_{P \beta 4}\right)$.

Figure 13(h). Transfer function for separated flow. $\beta=f_{\beta}\left(C_{P \alpha 4}, C_{P \beta 4}\right)$.

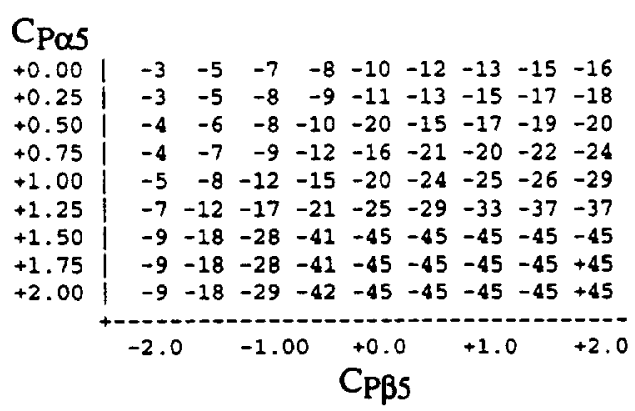

Figure 13(i). Transfer function for separated flow. $\alpha=f_{\alpha}\left(C_{P \alpha 5}, C_{P \beta 5}\right)$.

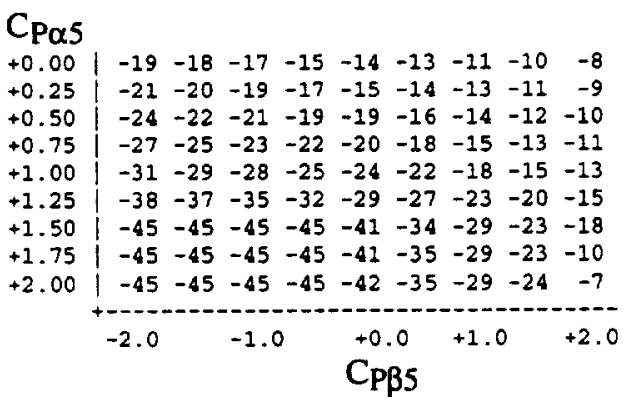

Figure 13(j). Transfer function for separated flow. $\beta=f_{\beta}\left(C_{P \alpha 5}, C_{P \beta 5}\right)$.

Figure 13(k). Transfer function for separated flow. $\alpha=f_{\alpha}\left(C_{P \alpha 6}, C_{P \beta 6}\right)$.

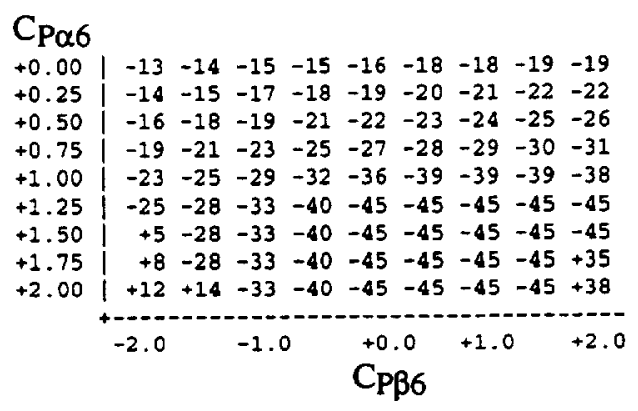

Figure 13I(I). Transfer function for separated flow. $\beta=f_{\beta}\left(C_{P \alpha 6}, C_{P \beta 6}\right)$. 


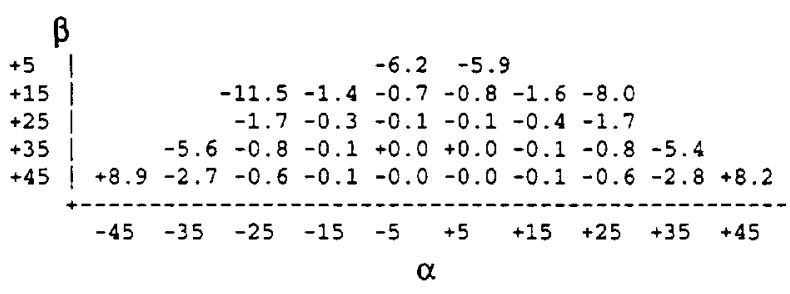

Figure 14(a). Static pressure coefficients, $C_{P S 1}$.

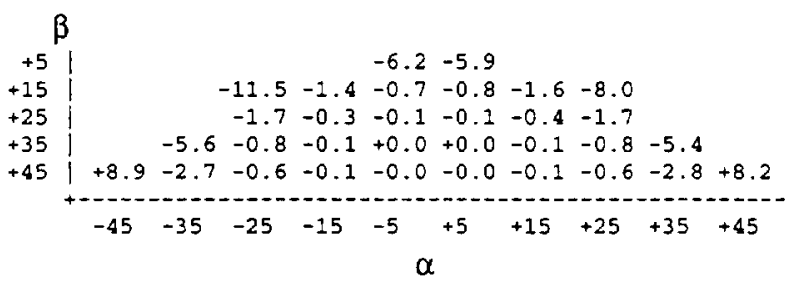

Figure 14(b). Total pressure coefficients, $C_{P+1}$.

$$
\begin{aligned}
& \beta
\end{aligned}
$$

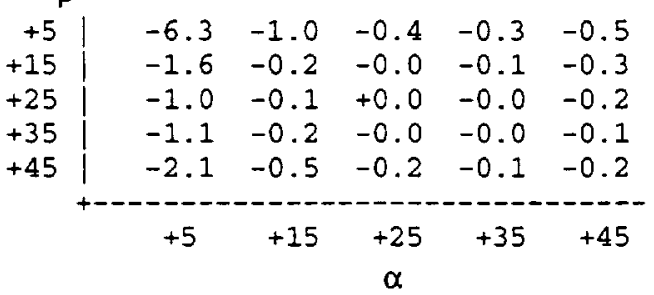

Figure 14(c). Static pressure coefficients, sector 2.

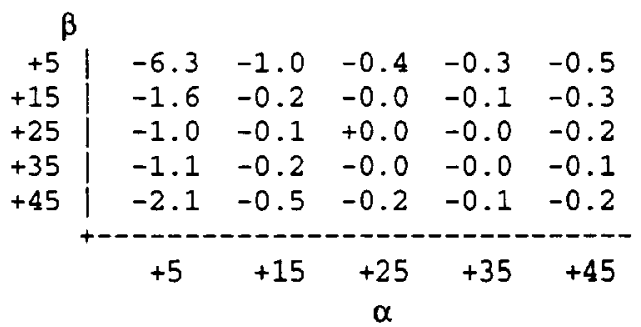

Figure $14(d)$. Total pressure coefficients, sector 3.

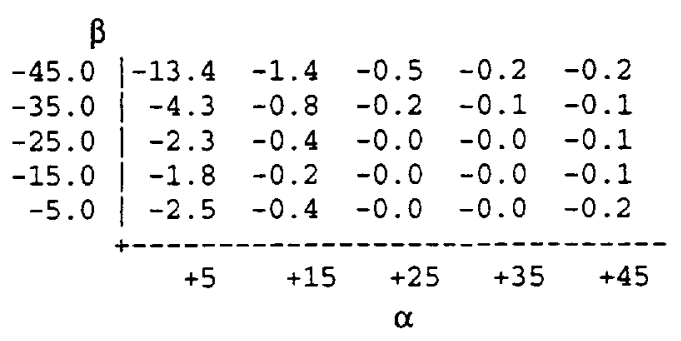

Figure 14(e). Static pressure coefficients, sector 3.

$$
\begin{array}{c|rrrrr}
\beta & \multicolumn{1}{c}{0} \\
-45.0 & -13.4 & -1.4 & -0.5 & -0.2 & -0.2 \\
-35.0 & -4.3 & -0.8 & -0.2 & -0.1 & -0.1 \\
-25.0 & -2.3 & -0.4 & -0.0 & -0.0 & -0.1 \\
-15.0 & -1.8 & -0.2 & -0.0 & -0.0 & -0.1 \\
-5.0 & -2.5 & -0.4 & -0.0 & -0.0 & -0.2 \\
+ & +5.0+15.0+25.0 & +35.0 & +45.0 \\
& \alpha
\end{array}
$$

Figure 14(f). Total pressure coefficients, sector 3.

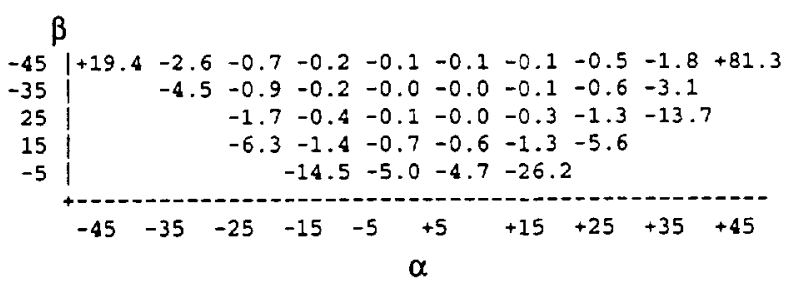

Figure 14(g). Static pressure coefficients, sector 4.

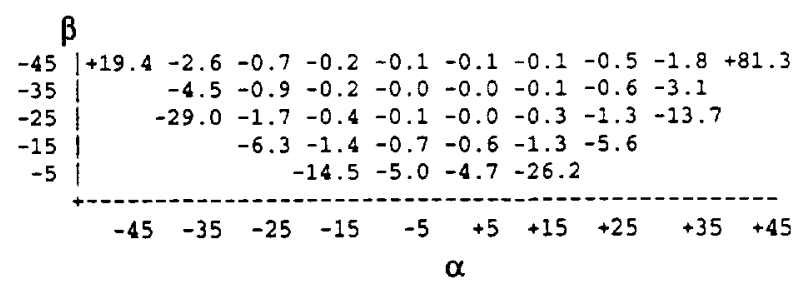

Figure 14(h). Total pressure coefficients, sector 5. 


$$
\begin{array}{c|ccccc}
\beta & & & & \\
-45.0 & -0.2 & -0.1 & -0.2 & -0.5 & -1.3 \\
-35.0 & -0.2 & -0.1 & -0.1 & -0.2 & -0.9 \\
-25.0 & -0.2 & -0.0 & -0.0 & -0.1 & -0.8 \\
-15.0 & -0.3 & -0.1 & -0.1 & -0.3 & -1.5 \\
-5.0 & -0.5 & -0.3 & -0.3 & -0.9 & -4.5 \\
& +-45.0 & -35.0 & -25.0 & -15.0 & -5.0
\end{array}
$$

\begin{tabular}{|c|c|c|c|c|c|}
\hline \multicolumn{6}{|c|}{$\beta$} \\
\hline-45.0 & -0.2 & -0.1 & -0.2 & -0.5 & -1.3 \\
\hline-35.0 & -0.2 & -0.1 & -0.1 & -0.2 & -0.9 \\
\hline-25.0 & -0.2 & -0.0 & -0.0 & -0.1 & -0.8 \\
\hline-15.0 & -0.3 & -0.1 & -0.1 & -0.3 & -1.5 \\
\hline-5.0 & -0.5 & -0.3 & -0.3 & -0.9 & -4.5 \\
\hline & -45.0 & -35.0 & -25.0 & -15.0 & -5.0 \\
\hline
\end{tabular}

Figure 14(i). Static pressure coefficients, sector 5.

Figure 14(j). Total pressure coefficients, sector 5.

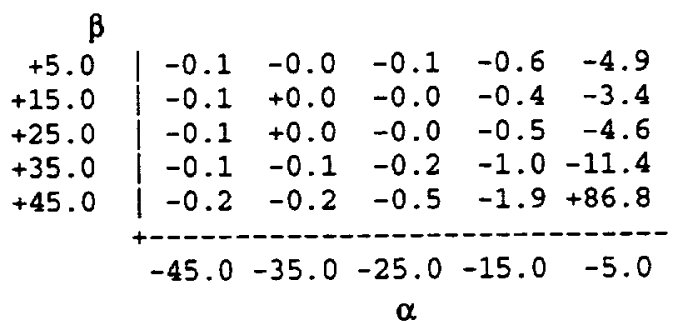

Figure 14(k). Static pressure coefficients, sector 6.

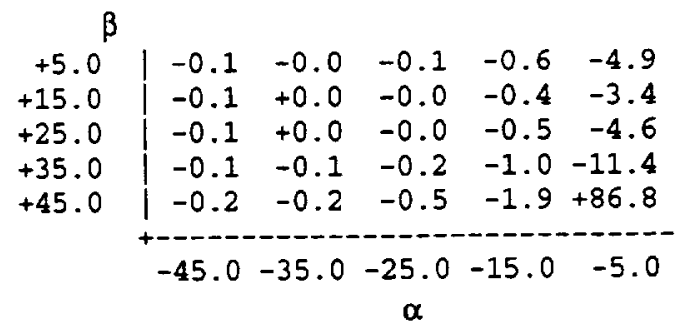

Figure $14 I(I)$. Total pressure coefficients, sector 6. 


\section{Appendix B-Calibration Code (Microsoft QuickBasic)}

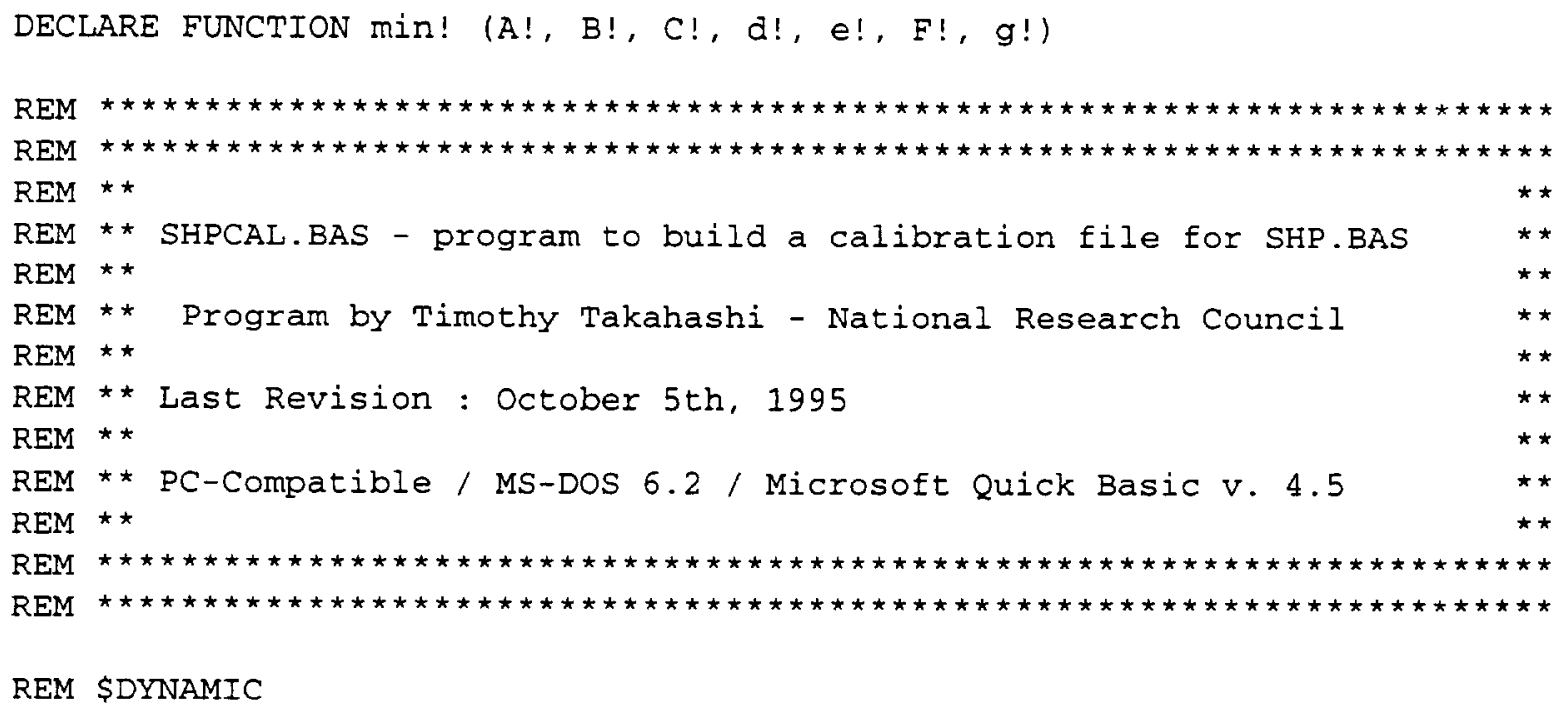




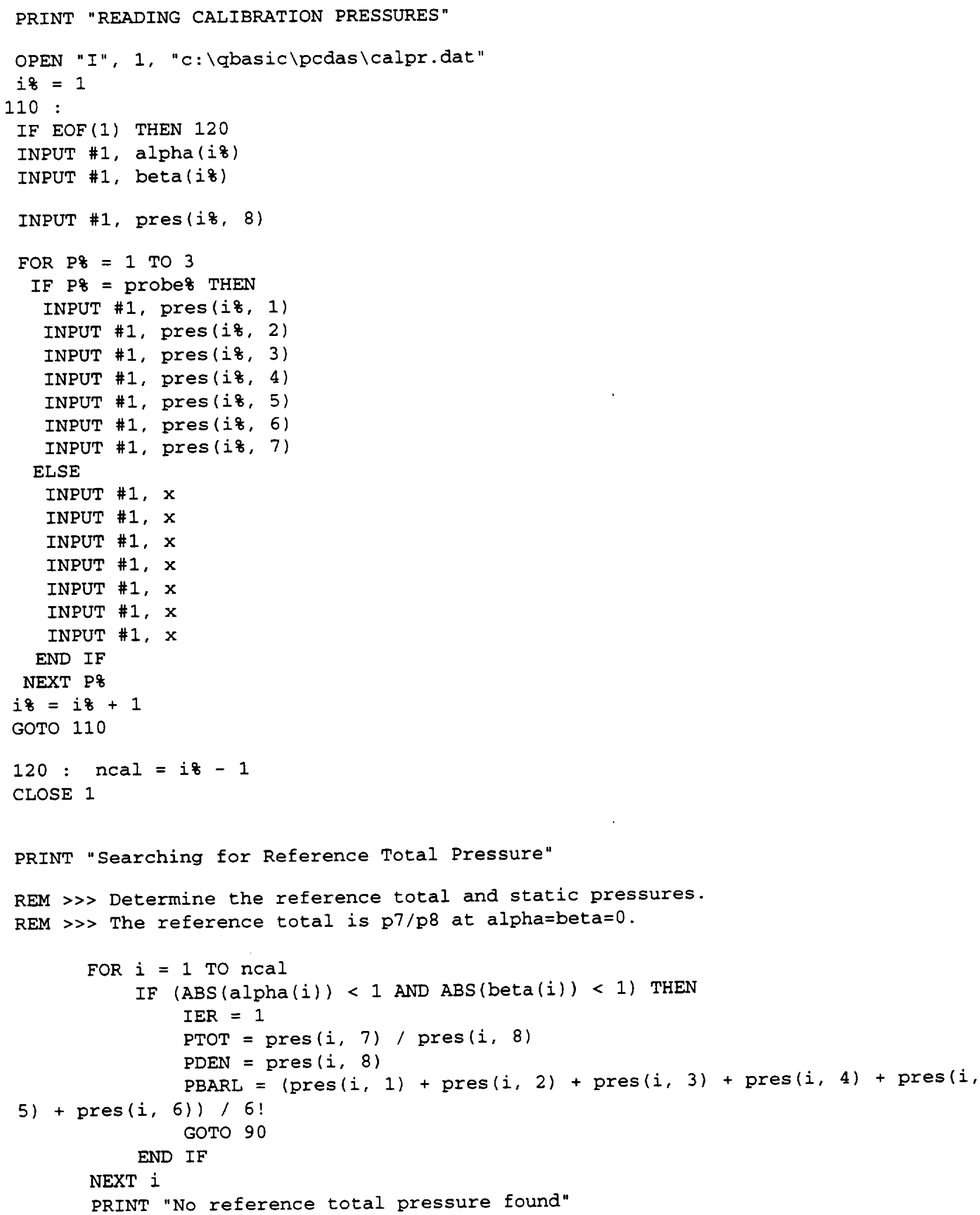


STOP

$90:$

PRINT "REFERENCE TOTAL PRESSURE = "; PTOT * PDEN; "PSI"

PST $=0$

PSTAT $=$ PST $/$ PDEN

REM $\gg>$ Compute Calibration Coefficients

PRINT "Computing Coefficients"

FOR $i=1$ TO ncal

REM >> non-dimensionalize pressures

FOR $j=1$ TO 8 $\operatorname{NDpres}(j)=\operatorname{pres}(i, j) / \operatorname{pres}(i, 8)$

NEXT $j$

$i x \frac{8}{8}=($ alpha(i) $) / 5+10$

iyo $=(\operatorname{beta}(i)) / 5+10$

REM $\gg$ Check to make sure pressure at hole 7 is not the lowest

REM $\gg>$ pressure (Second check for separation at hole 7).

PMIN $=\min ($ NDpres (1), NDpres (2), NDpres (3), NDpres (4), NDpres (5) ,

NDpres (6), NDpres (7))

IF (NDpres $(7)=$ PMIN) THEN 150

REM $\gg$ Compute the Coefficients for each sector

REM >> Zone 1 .

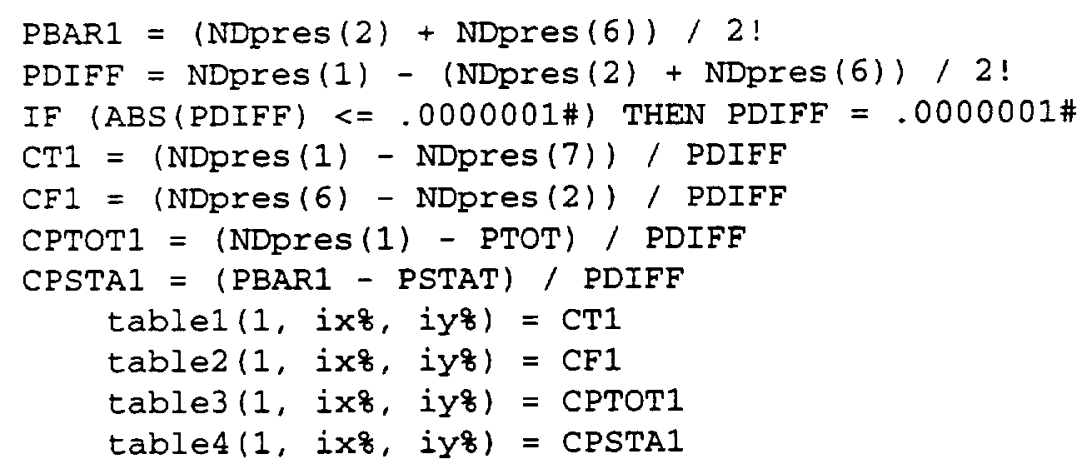

REM $\gg>$ Zone 2 .

PDIFF $=$ NDpres (2) - (NDpres (1) + NDpres (3)) $/ 2$ !

PBAR2 $=(\operatorname{NDpres}(1)+\operatorname{NDpres}(3)) / 2$ !

IF (ABS (PDIFF) $<=.0000001 \#)$ THEN PDIFF $=.0000001 \#$

$\mathrm{CT} 2=($ NDpres $(2)-$ NDpres(7) $) /$ PDIFF

$\mathrm{CF} 2=($ NDpres $(1)-$ NDpres(3)) / PDIFF

CPTOT2 = (NDpres (2) - PTOT) $/$ PDIFF

CPSTA2 = (PBAR2 - PSTAT $) /$ PDIFF

table1 (2, ixz, iyz) = CT2

table2 (2, ix $\left.8, i y^{8}\right)=C F 2$ 
table3 (2, ixz, iyz) = CPTOT2

table4 $(2$, ixz, iy $)=$ CPSTA 2

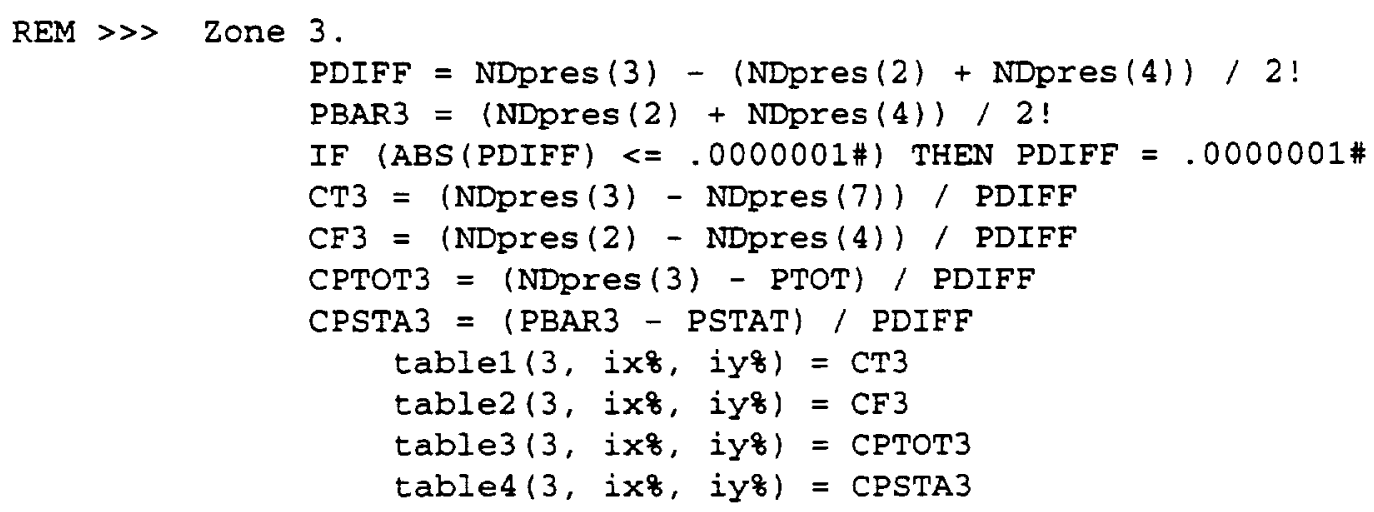

REM $\gg$ Zone 4 .

PDIFF $=$ NDpres (4) - (NDpres (3) + NDpres (5)) / 2 !

PBAR4 $=($ NDpres $(3)+$ NDpres $(5)) / 2$ !

IF (ABS (PDIFF) $<=.0000001 \#)$ THEN PDIFF $=.0000001 \#$

CT4 = (NDpres(4) - NDpres(7)) / PDIFF

$\mathrm{CF} 4=($ NDpres $(3)$ - NDpres (5)) / PDIFF

CPTOT4 $=($ NDpres $(4)-$ PTOT $) /$ PDIFF

CPSTA4 = (PBAR4 - PSTAT) $/$ PDIFF

table1 (4, ixz, iyz) = CT4

table2 (4, ixz, $\left.i y^{8}\right)=C F 4$

table3 (4, ixf, iYz) = CPTOT4

table4 (4, ixz, iYz) = CPSTA 4

REM $\gg$ Zone 5 .

PDIFF $=$ NDpres $(5)-($ NDpres $(4)+\operatorname{NDpres}(6)) / 2$ !

PBAR5 $=($ NDpres $(4)+$ NDpres $(6)) / 2$ !

IF (ABS (PDIFF) $<=.0000001 \#$ ) THEN PDIFF $=.0000001 \#$

CT5 $=($ NDpres(5) - NDpres(7)) / PDIFF

CF5 $=($ NDpres $(4)-$ NDpres $(6)) /$ PDIFF

CPTOT5 = (NDpres $(5)-$ PTOT) $/$ PDIFF

CPSTA5 = (PBAR5 - PSTAT $) /$ PDIFF

table1 (5, ix8, iY8) = CT5

table2 (5, ixz, iy $)=C F 5$

table3 (5, ix8, iyz) = CPTOT5

table4 (5, ixz, iyz) = CPSTA5

REM $\gg$ Zone 6 .

PDIFF $=$ NDpres (6) - (NDpres (5) + NDpres (1)) / 2 !

PBAR6 $=(\operatorname{NDpres}(5)+\operatorname{NDpres}(1)) / 2$ !

IF (ABS (PDIFF) $<=.0000001 \#)$ THEN PDIFF $=.0000001 \#$

CT6 $=($ NDpres $(6)-$ NDpres (7) $) /$ PDIFF

CF6 $=($ NDpres (5) - NDpres(1)) / PDIFF

CPTOT6 $=(\operatorname{NDpres}(6)-$ PTOT $) /$ PDIFF

CPSTA6 = (PBAR6 - PSTAT) $/$ PDIFF

tablel (6, ix\%, iY 8$)=\mathrm{CT} 6$

table2 (6, ix8, iY8) = CF6

table3 (6, ix,$\left.i_{Y} 8\right)=$ CPTOT6

table4 $(6, i x 8, i y 8)=$ CPSTA 6 


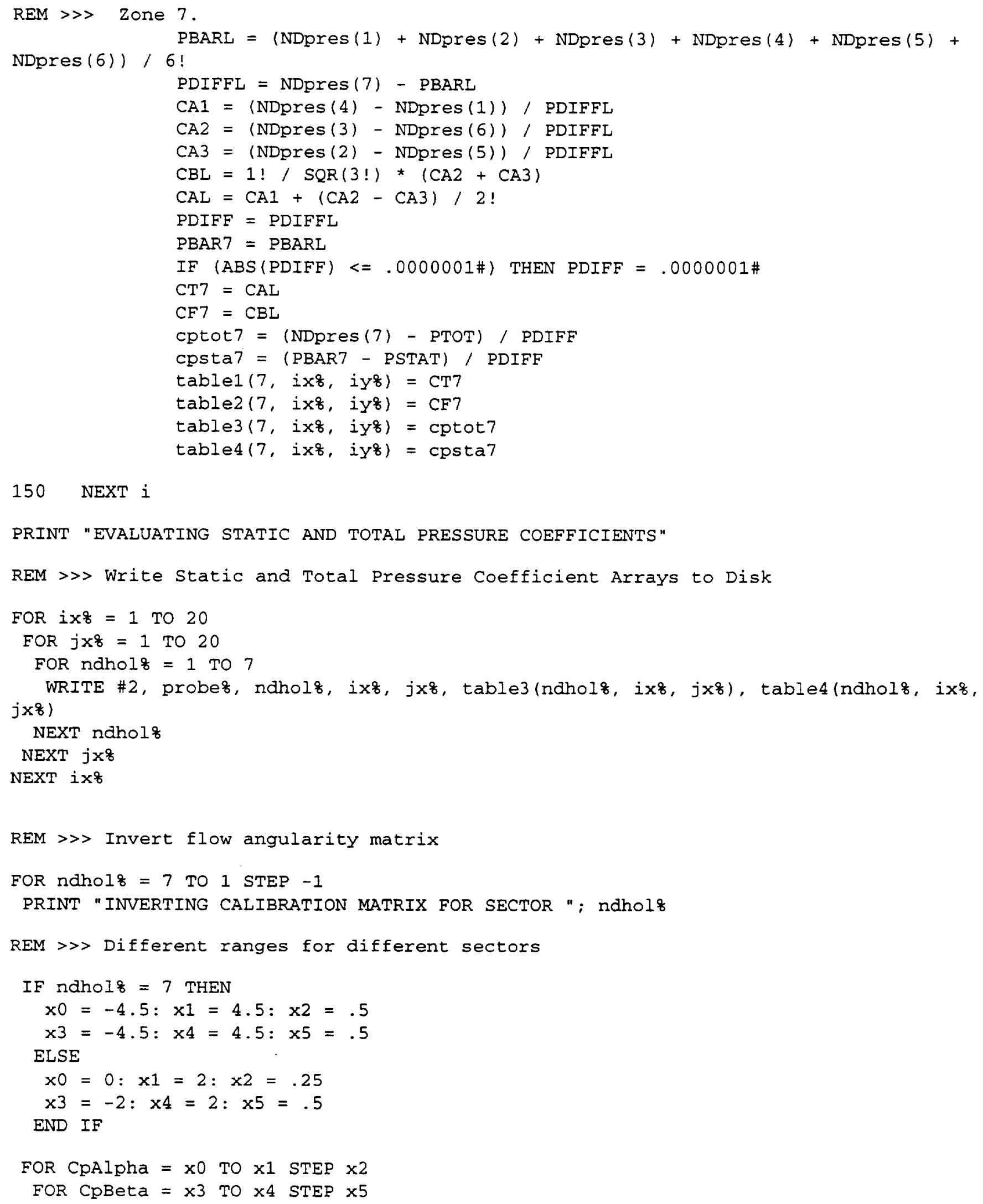


FOR $j 3=j 2-.25$ TO j2 32.25 STEP .0625

$$
i Y z=\operatorname{INT}(i 3): i z=i 3-i Y^{8}
$$

$j Y^{8}=\operatorname{INT}(j 3): j z=j 3-j Y^{8}$

$A=$ tablel (ndholq, $\left.i y^{q}+1, j y^{q}+1\right) * i z * j z+t a b l e 1$ (ndhol $\left.8, i y^{q}, j y^{q}+1\right) *(1$

$-i z) * j z+$ tablel (naholo, $\left.i y^{q}, j y z\right) *(1-i z) *(1-j z)+$ tablel (ndholz, $i y^{q}+$

$1, j y z) * i z \star(1-j z)$

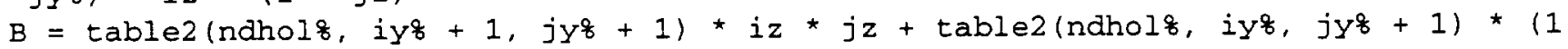

$-i z) \star j z+$ table2 (ndhol $8, i y 8, j y \&) *(1-i z) *(1-j z)+$ table2 (ndhol\%, iyz +

$1, j y z) * i z *(1-j z)$

$$
\begin{aligned}
& e=(A-\text { CpAlpha }) \wedge 2+(B-\text { CpBeta }) \wedge 2 \\
& I F e<e 0 \text { THEN } 14=i 3: j 4=j 3: e 0=e
\end{aligned}
$$

NEXT $j 3$

NEXT i3

alpha $=(14-10) * 5$

beta $=(j 4-10) * 5$

PRINT USING "SECTOR \# | CPALPHA +\#.\#\# CPBETA +\#.\#\#\#| ALPHA +\#\#.\# BETA +\#\#.\#"; ndhol\%; CpAlpha; CpBeta; alpha; beta

IF eO > I THEN BEEP: alpha = -999: beta $=-999:$ GOTO 1001

$1001:$

WRITE \#2, probeq, ndholz, CpAlpha, CpBeta, alpha, beta NEXT CpBeta

NEXT CPAlpha

NEXT ndhol\%

PRINT

NEXT probeq

CLOSE

END

REM \$STATIC

FUNCTION min (A, B, C, d, e, F, g)

$\mathrm{n}=0$

IF $\mathrm{A}<\mathrm{B}$ THEN $\mathrm{n}=\mathrm{A}$ ELSE $\mathrm{n}=\mathrm{B}$

IF $C<n$ THEN $n=C$

IF $\mathrm{d}<\mathrm{n}$ THEN $\mathrm{n}=\mathrm{d}$

IF $e<n$ THEN $n=e$

IF $\mathrm{F}<\mathrm{n}$ THEN $\mathrm{n}=\mathrm{F}$

IF $\mathrm{g}<\mathrm{n}$ THEN $\mathrm{n}=\mathrm{g}$

$\min =\mathrm{n}$

END FUNCTION 



\section{Apendix C-Data Acquisition Code (Microsoft QuickBasic)}

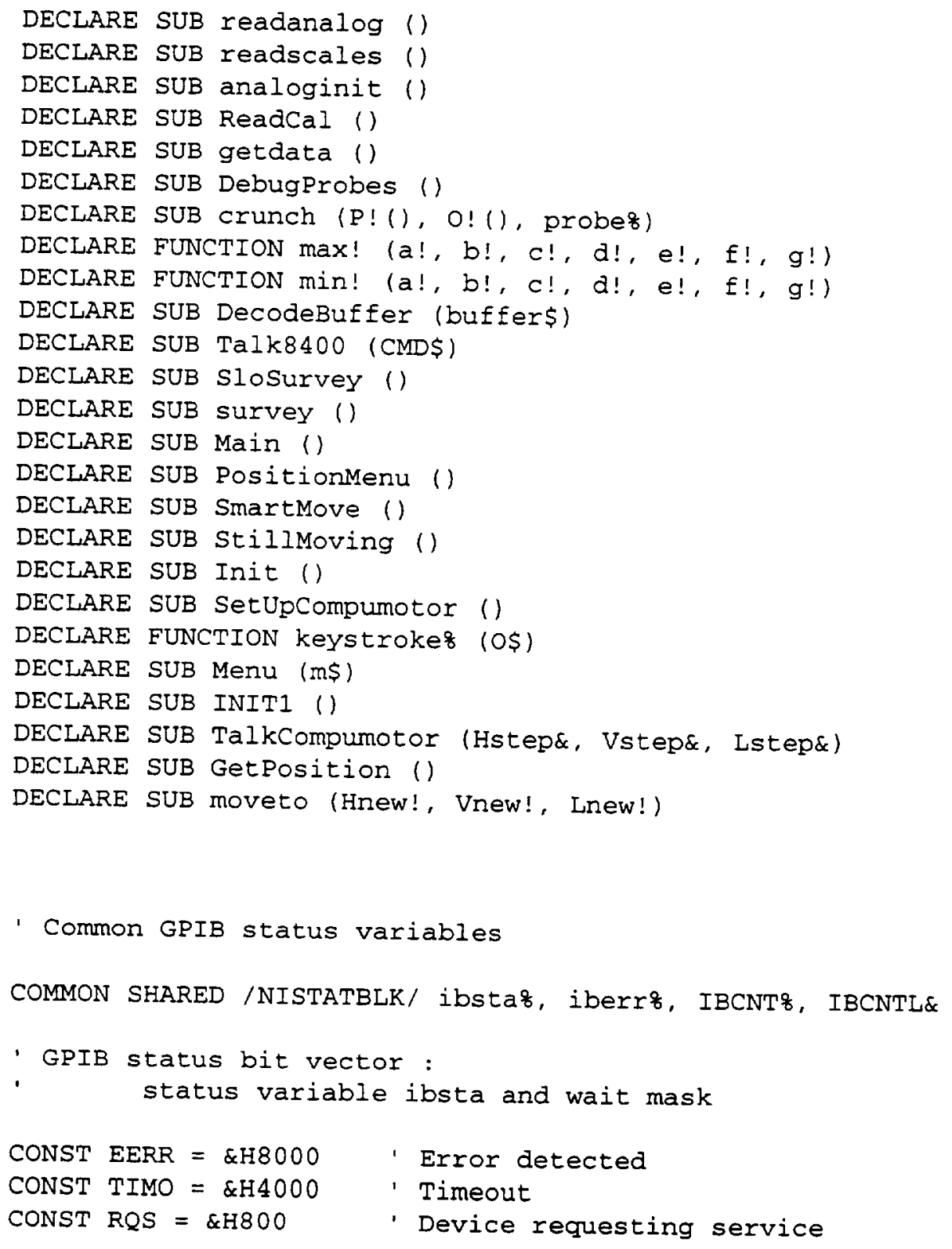




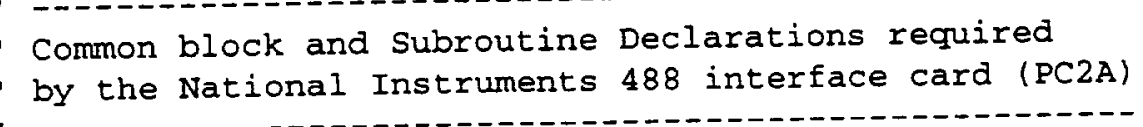

DIM SHARED psiq, spxo, echo\%

DIM SHARED Nvals, PktInfo (19), pktmsg\$, pktXdat (4096)

DIM SHARED buffer $3 \$, x \$$

DIM SHARED volts (16), scale(10)

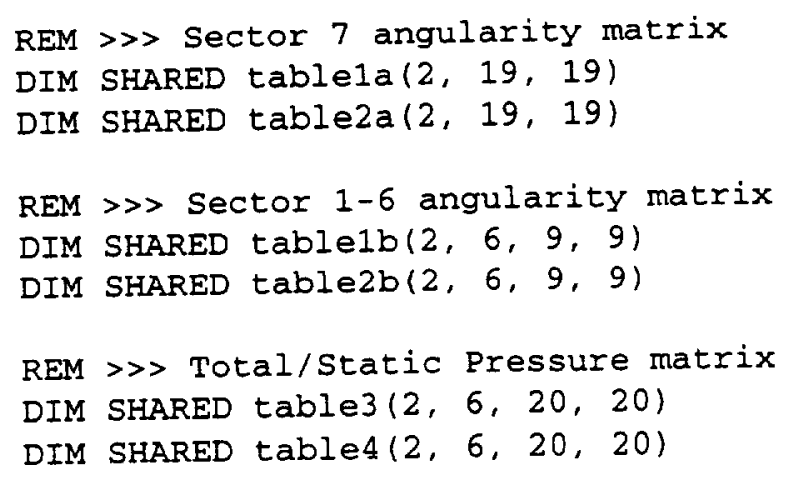




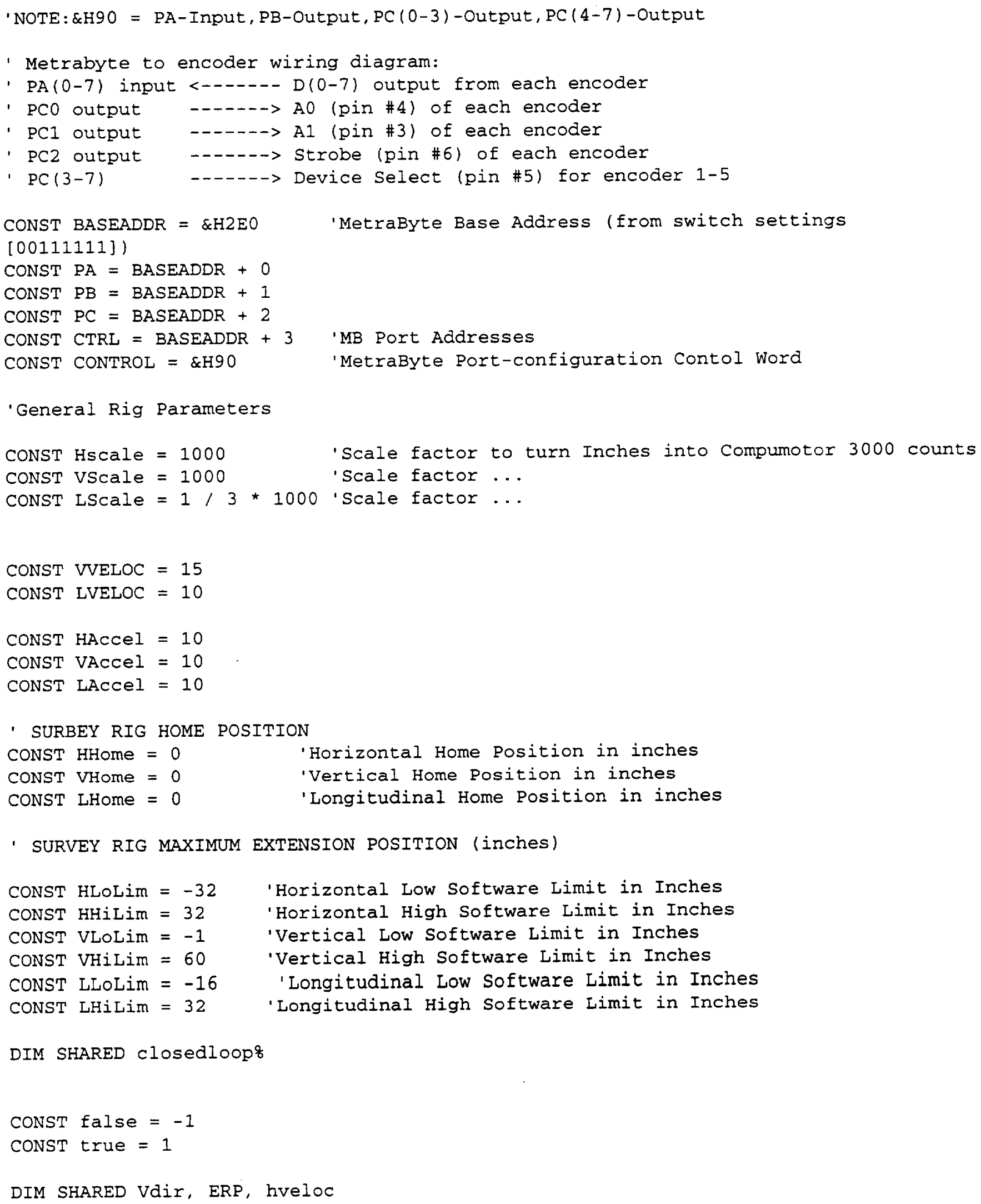


CALL Init

CALL ReadCal

CALL SetUpCompumotor

CALL Main

CHDIR "c: \qbasic \pcdas"

RUN "MENU. BAS"

SUB analoginit

DIM param8 (60), DAT\&(32000), gainz(15), nn(15), pp(15), offset (15)

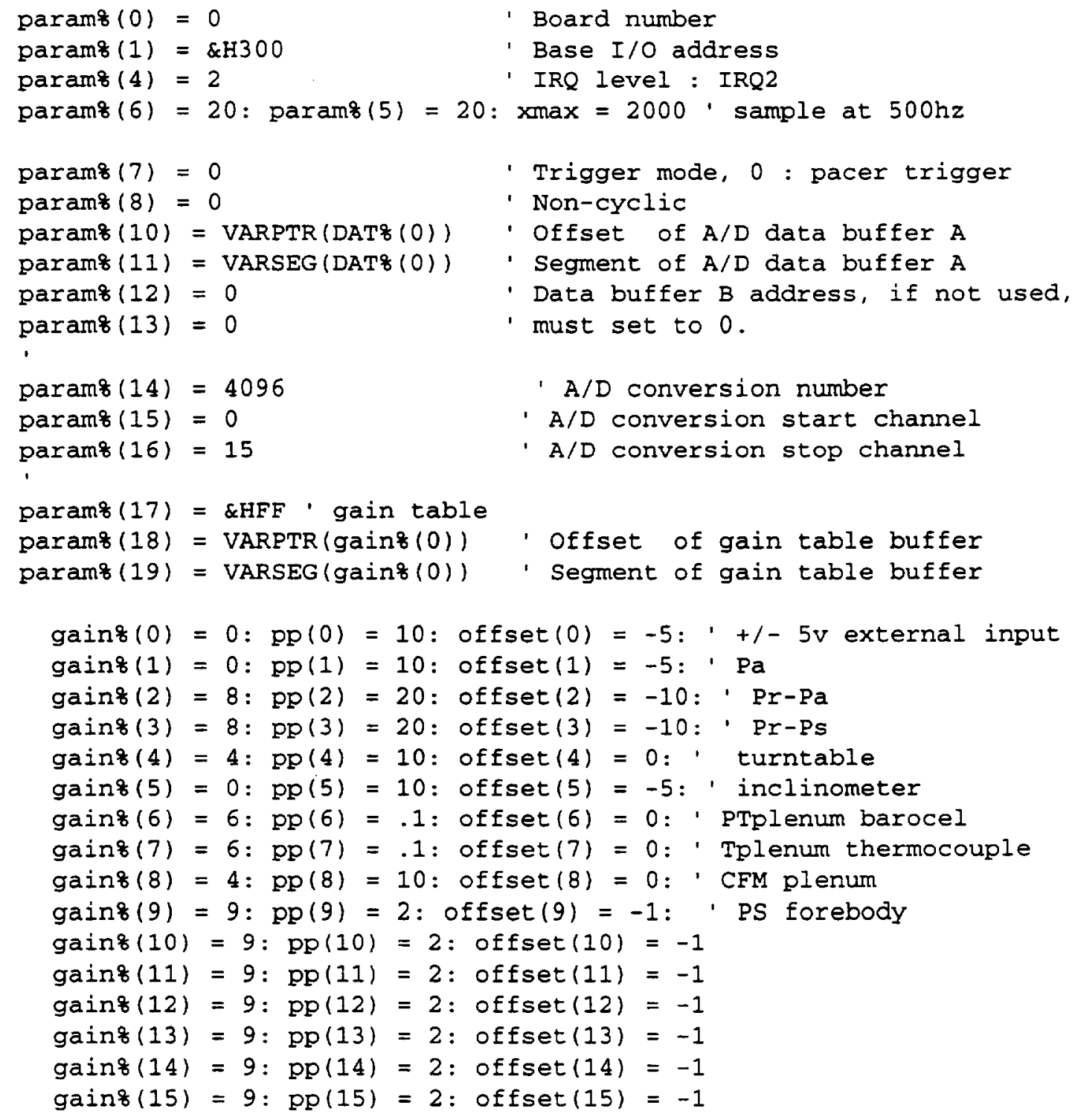




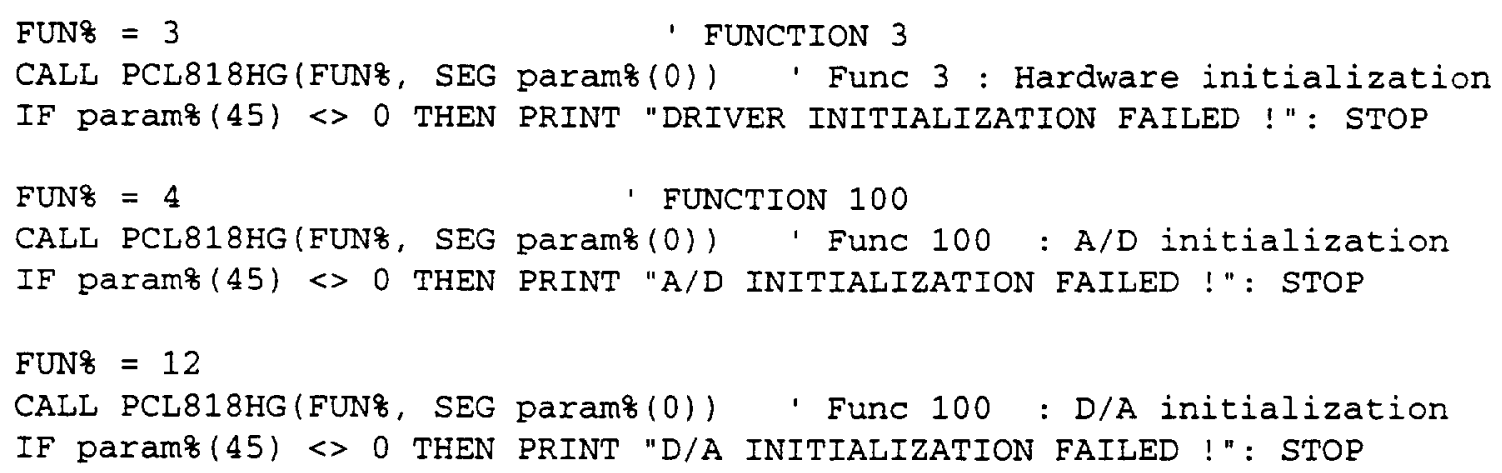




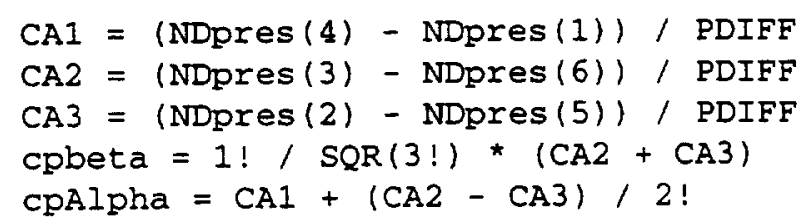

REM Check to see if flow angularity is outside zone 7 calibration range IF cPAlpha $>4.5$ OR cpAlpha < -4.5 OR cpbeta $>4.5$ OR cpbeta < -4.5 THEN 21

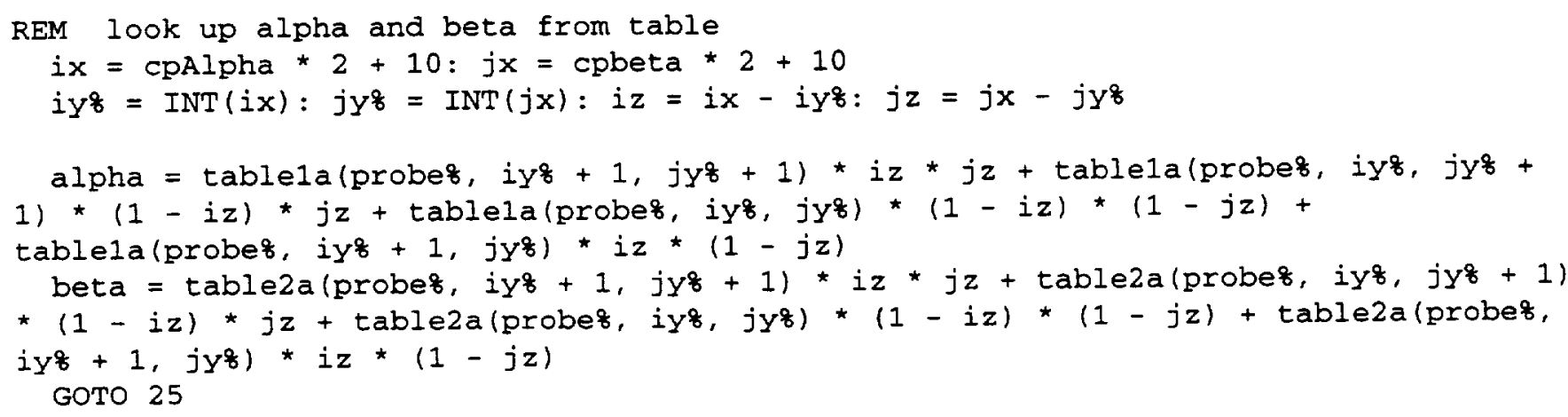




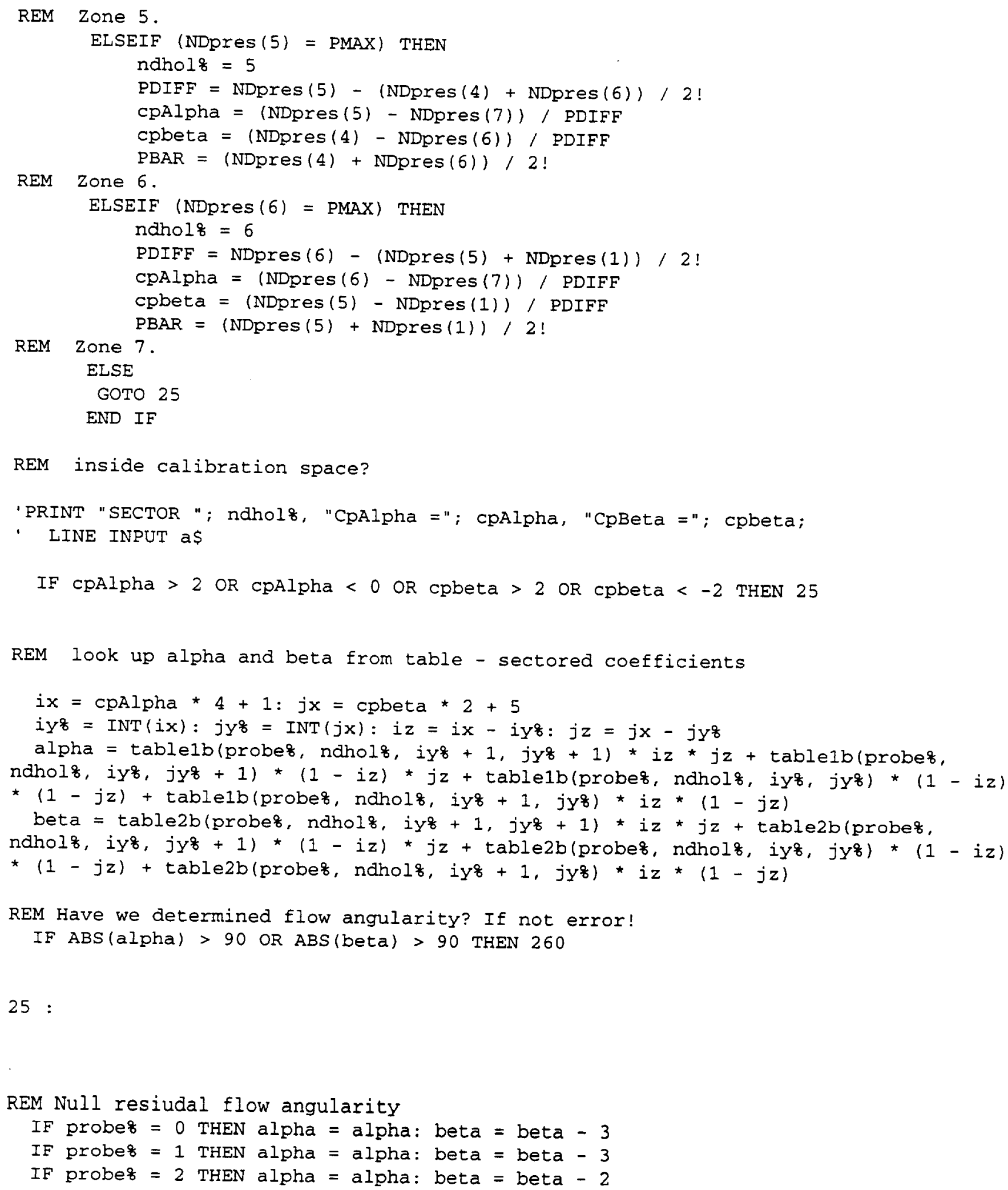

REM Determine Static and Total Pressure Coefficients 


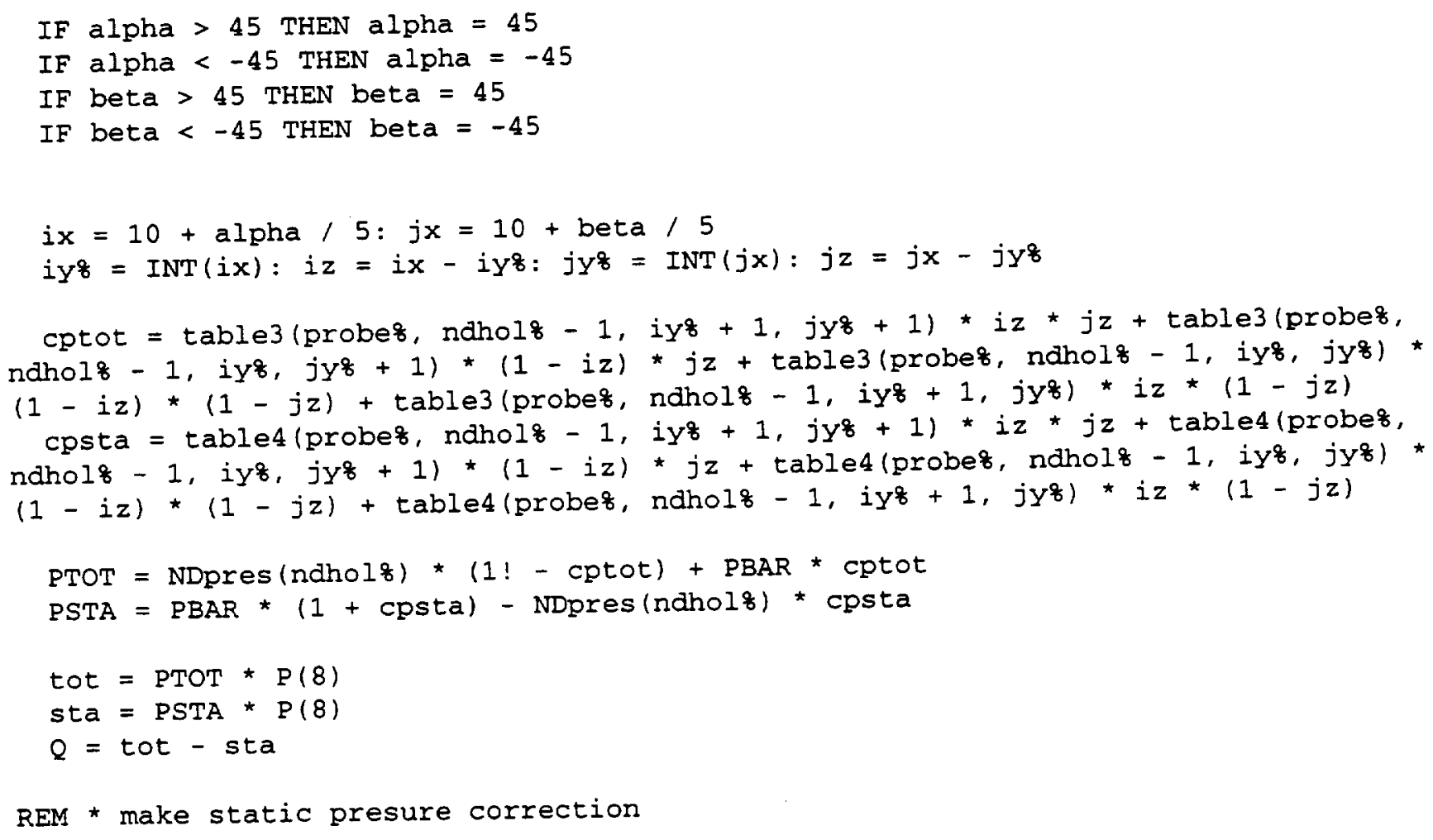


END SUB

SUB DebugProbes

SCREEN 0: WIDTH 80, 43

CLS

PRINT "PROBE DEBUG -

PRINT

PRINT " PROBE PRESSURE"

PRINT

PRINT

VIEW PRINT 5 TO 43

Talk8400 ("ODO")

$1101:$

SOUND 1000,1

Talk8400 ("AD1 11 ")

$\operatorname{tmx}=$ TIMER +1

11102 : IF TIMER < tmx THEN 11102

Talk8400 ("OD1 1")

LOCATE 5,1

FOR i $=1$ TO 26

PRINT USING " \#\#\# +\#\#\#.\#\# psf "; i8; pktXdat(i8) * 144;

$n q=$ pktXdat $(i q) * 144 / 2$

IF $\mathrm{n} 8>40$ THEN $\mathrm{n} 8=40$

IF $n \&<-40$ THEN $n \&=40$

IF $\mathrm{n} 8>0$ THEN PRINT STRINGS( $\mathrm{n} 8, " \# ") ; \operatorname{TAB}(78) ; "$;

IF $n \&<=0$ THEN PRINT STRING\$ $(-n 8, " x ") ; \operatorname{TAB}(78) ; "$ "

IF $i \%=1$ OR $i q=2$ OR $i q=9$ OR $i q=16$ OR $i q=23$ THEN PRINT STRING $\left(70, "{ }^{\prime}=1\right)$

NEXT iq

IF INKEY\$ = " " THEN 1101

CALL ibonl (psiz, 0): psiz = 0

LINE INPUT "PRESS ENTER TO RETURN TO MAIN MENU"; a\$

VIEW PRINT 1 TO 43

SCREEN 0: WIDTH 80,43

CLS

END SUB 







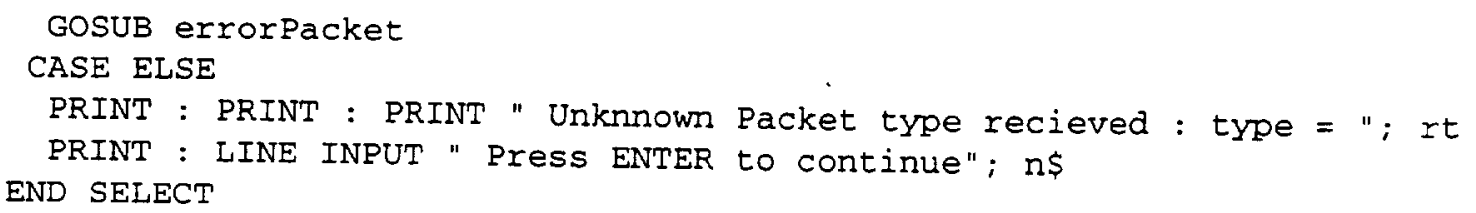




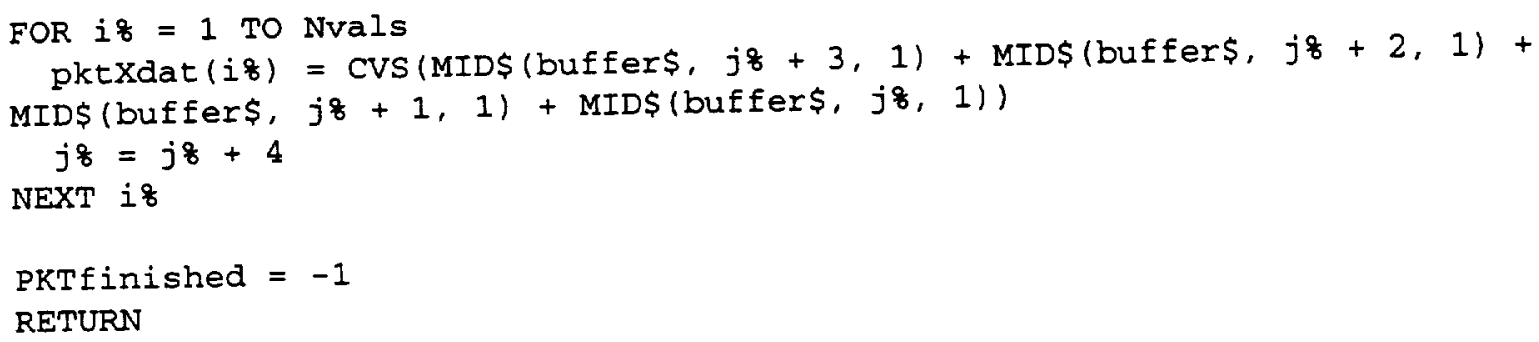




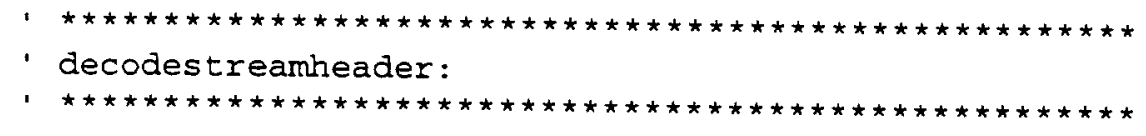

DecodestreamHeader :

'

' we have already decoded response code, response type,

- and response length

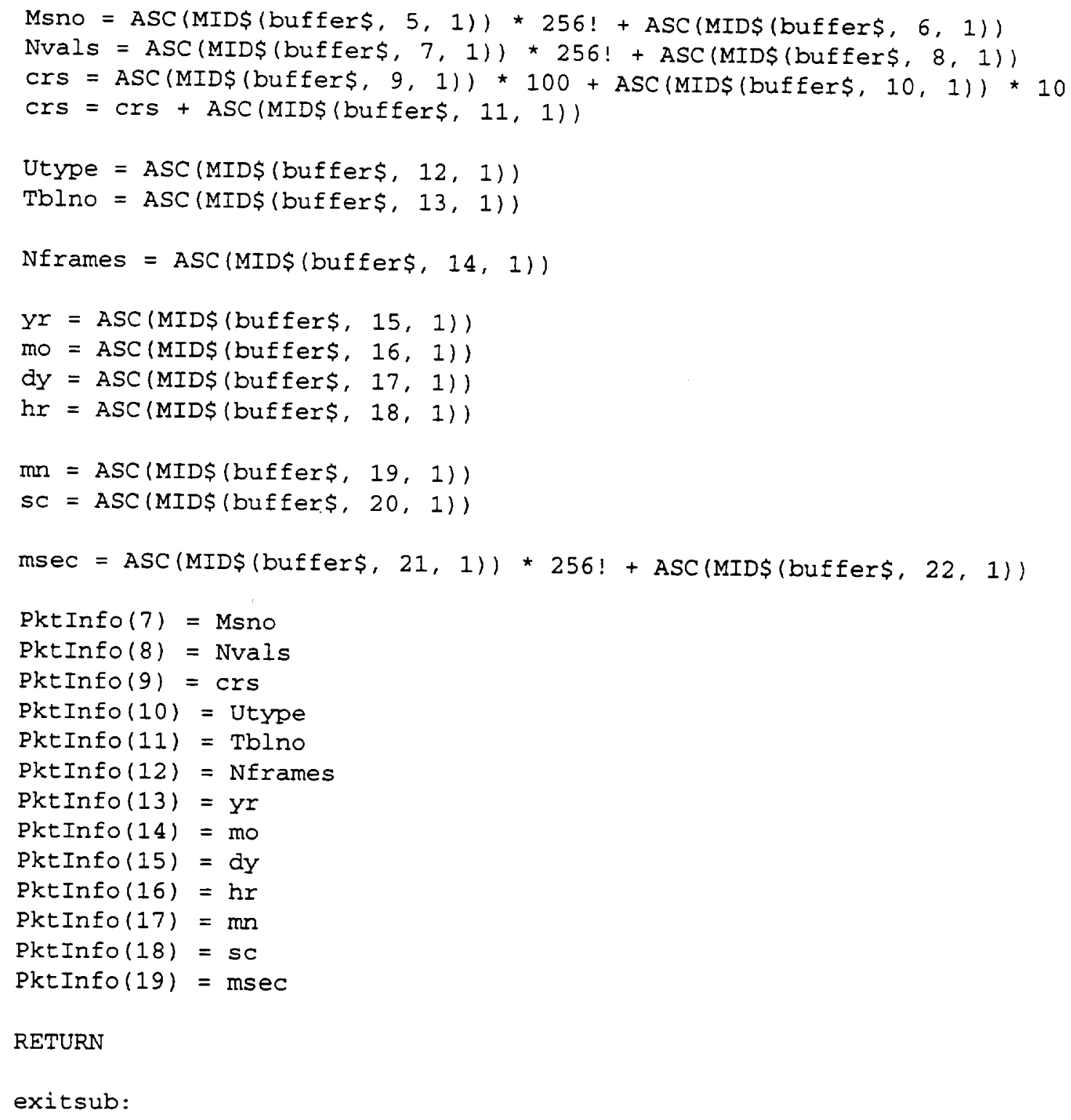


'Parallel Read Encoder Position for MetraByte PIO-12 and AR Absolute 'encoders. The ACT array is used to determine which encoders will be 'Read. The output will be in the arrays ERR and PLC. The method ' used to reduce the parallel data will be determined by the status of ' the SP parameter for each encoder (either Binary or BCD).

- PARALLEL READ ENCODER POSITION

DIM plc(4)

'Set-up's for MetraByte Parallel Ports

- Converse in parallel

OUT CTRL, CONTROL'set up MetraByte

$E R P=$ false

FOR RO = 0 TO 4

IF $A C T(R \&)<0$ THEN

$\mathrm{DEV}=2 \wedge(\mathrm{R} 8+3) \cdot$ Select which encoder to poll for position and error data

OUT PC, $4+\mathrm{DEV}+3$ 'Strobe High (4), AO and AI High (3), Device Selected

OUT PC, DEV +3 'Strobe Low, etc.

FOR is = 0 TO delay: NEXT is

$L S B=I N P(P A) \cdot$ Get LSB when $A 0$ and $A 1$ high

OUT PC, DEV + 1'Drop A1 LOW

FOR iz = 0 TO delay: NEXT if

LSB2 = INP(PA) ' Get LSB2 when AO high and A1 low

OUT PC, DEV 'Drop AO LOW as well

FOR if = 0 TO delay: NEXT if

MSB2 = INP(PA) ' Get MSB2 when AO and A1 low

OUT PC, DEV + 2'Raise AI high

FOR if = 0 TO delay: NEXT if

$M S B=I N P(P A) \quad$ Get MSB when $A O$ low and $A 1$ high

OUT PC, $4+$ DEV + 3 'Bring up all lines

'Convert data to position and error information

IF $\operatorname{ESB}(R 8,6)=$ "0" THEN

' Data interpreted as Binary

SIGN $=1:$ IF $($ LSB AND 16) = 16 THEN LSB = LSB - 16: SIGN $=-1$

IF (LSB AND 64) = 64 THEN ERP = true: LSB = LSB - 64

IF (LSB AND 128) = 128 THEN ERP = true: LSB = LSB - 128

$\mathrm{plc}(\mathrm{Rq})=((\mathrm{LSB} / 65536 !)+($ LSB2 $/ 512)+\mathrm{MSB} 2+(\mathrm{MSB} * 256)) *$ SIGN 


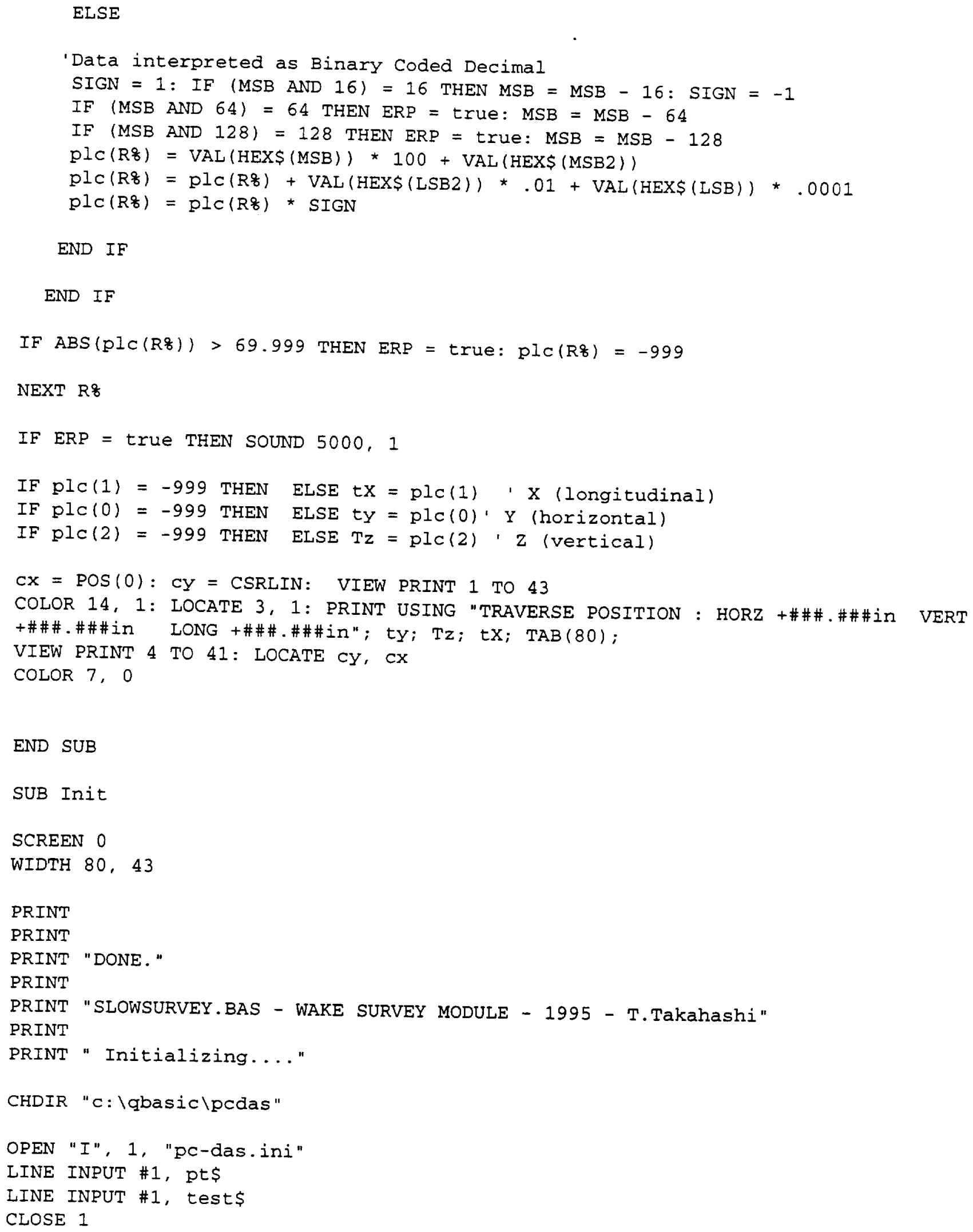


IF $\operatorname{RIGHT}(p t \$, 1)=" \backslash "$ AND RIGHT\$ $(p t \$, 2)<>": \backslash$ THEN pt\$ = LEFT\$(pt\$, LEN(pt\$) 1)

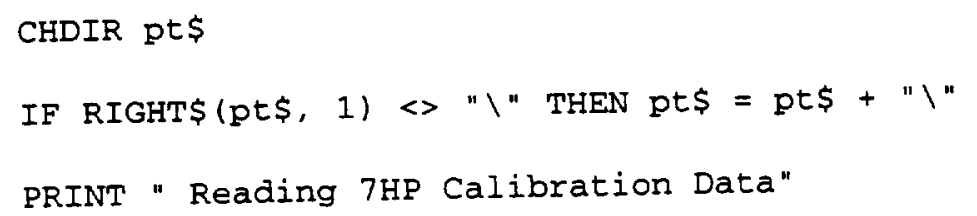


done:

\section{END SUB}

FUNCTION $\max (a, b, c, d, e, f, g)$

$\mathrm{n}=0$

IF $\mathrm{a}>\mathrm{b}$ THEN $\mathrm{n}=\mathrm{a}$ ELSE $\mathrm{n}=\mathrm{b}$

IF $c>n$ THEN $n=c$

IF $d>n$ THEN $n=d$

IF $e>n$ THEN $n=e$

IF $f>n$ THEN $n=f$

IF $\mathrm{g}>\mathrm{n}$ THEN $\mathrm{n}=\mathrm{g}$

$\max =n$

END FUNCTION

SUB Menu (m\$)

$\mathrm{CX}=\mathrm{POS}(0): \mathrm{CY}=\mathrm{CSRLIN}$

VIEW PRINT 1 TO 43

LOCATE 1, 1: COLOR 7, 1: PRINT TAB (37); "PC-DAS"; TAB (80);

LOCATE 2, 1:

flago $=32$

FOR if = 1 TO LEN (m\$)

IF flago $=32$ THEN COLOR 15, 7 ELSE COLOR 0,7

flago = ASC $(\operatorname{MID} \$(\mathrm{~m} \$, i q, 1))$

PRINT CHRS(flagz);

NEXT if

PRINT TAB $(80)$;

LOCATE 42,1 : COLOR 0,3

PRINT "PATH: "; pt\$; TAB(30); "TEST: "; test\$; TAB(60); TIME\$; " "; DATE\$; TAB (79);

VIEW PRINT 4 TO 41

IF $C Y<4$ THEN CY $=4$

LOCATE CY, CX

COLOR 7,0

END SUB

FUNCTION $\min (a, b, c, a, e, f, g)$

$n=0$

IF $\mathrm{a}<\mathrm{b}$ THEN $\mathrm{n}=\mathrm{a}$ ELSE $\mathrm{n}=\mathrm{b}$

IF $c<n$ THEN $n=c$

IF $\mathrm{d}<\mathrm{n}$ THEN $\mathrm{n}=\mathrm{d}$

IF $e<n$ THEN $\mathrm{n}=\mathrm{e}$

IF $f<n$ THEN $n=f$

IF $\mathrm{g}<\mathrm{n}$ THEN $\mathrm{n}=\mathrm{g}$

$\min =\mathrm{n}$ 


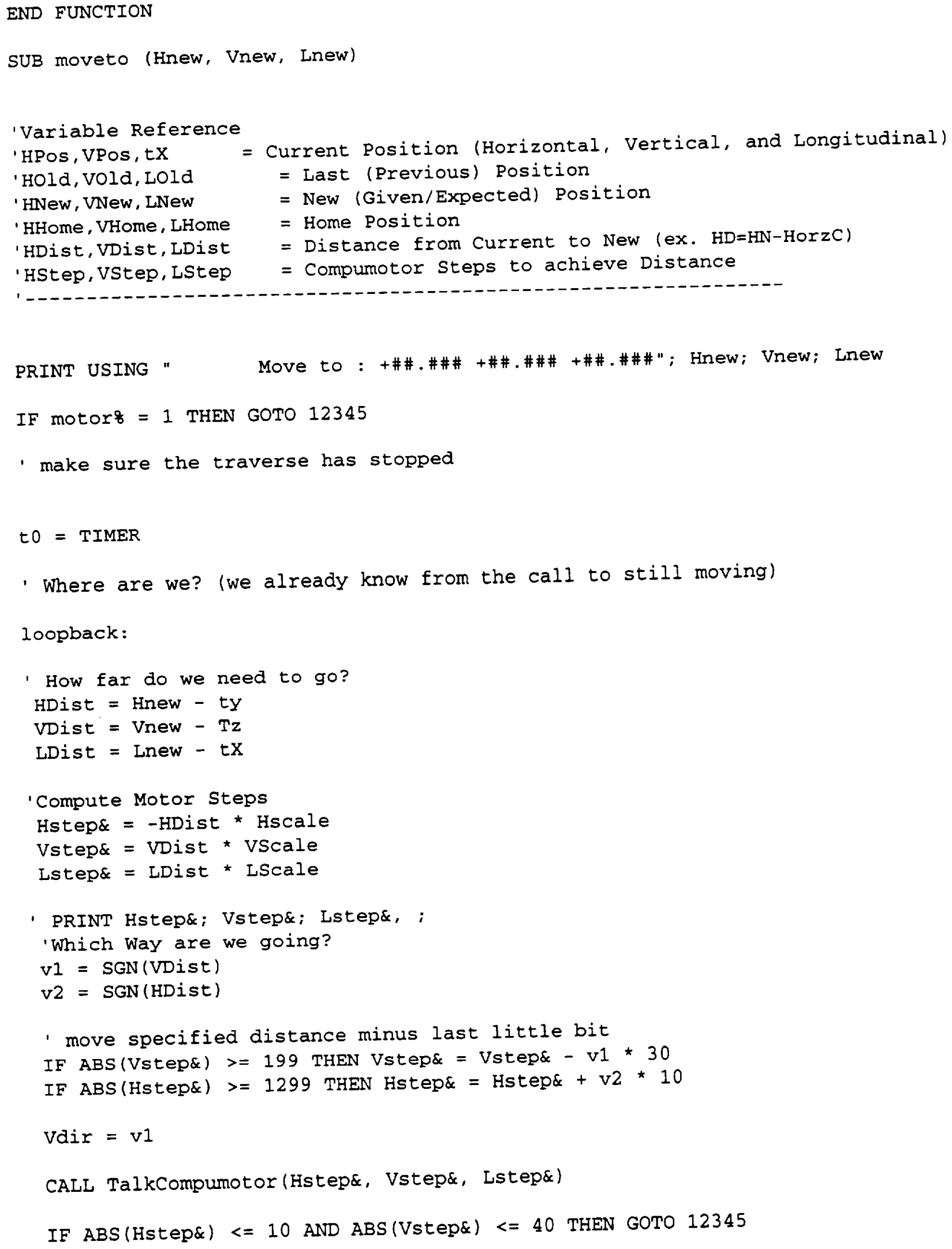




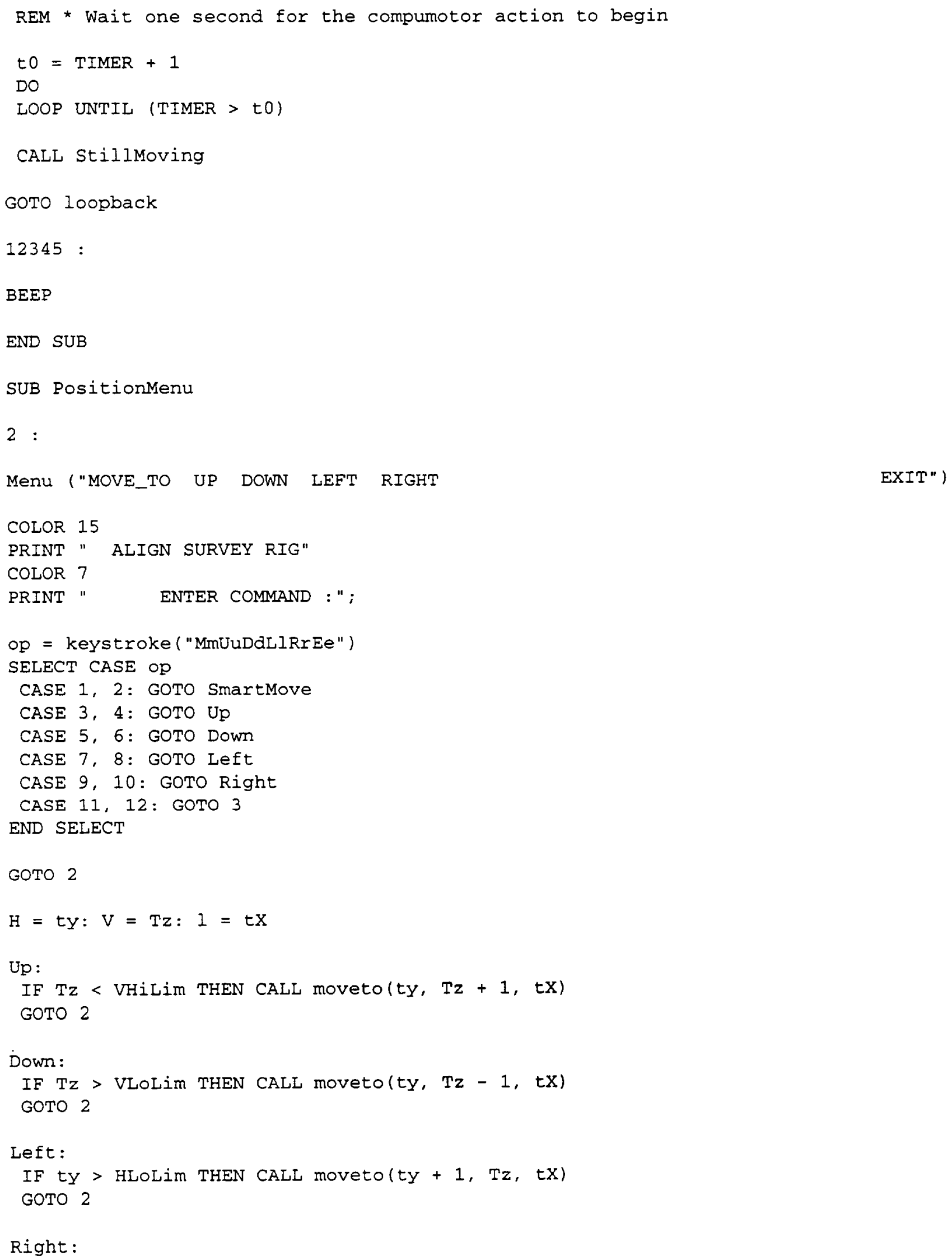

Right : 


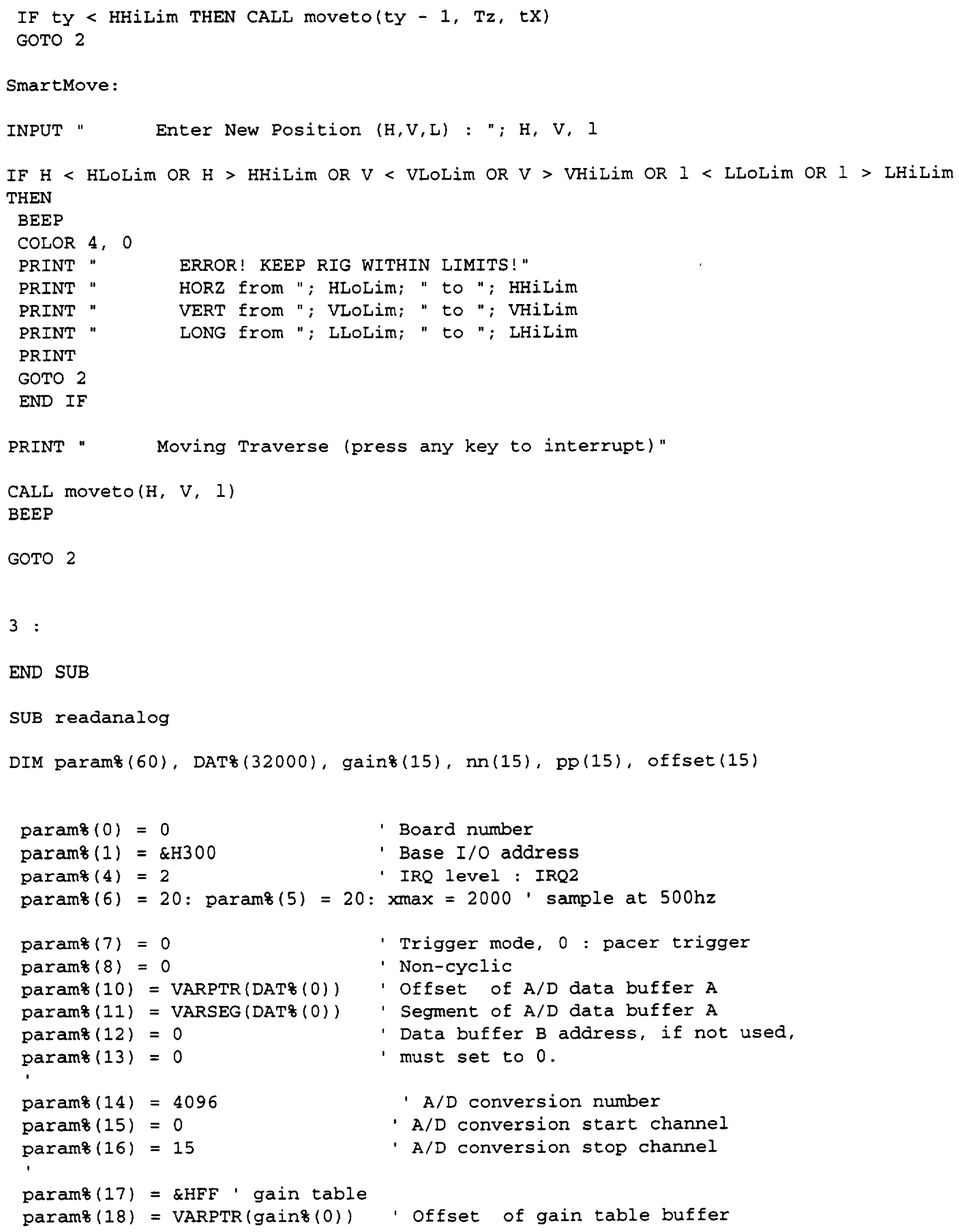




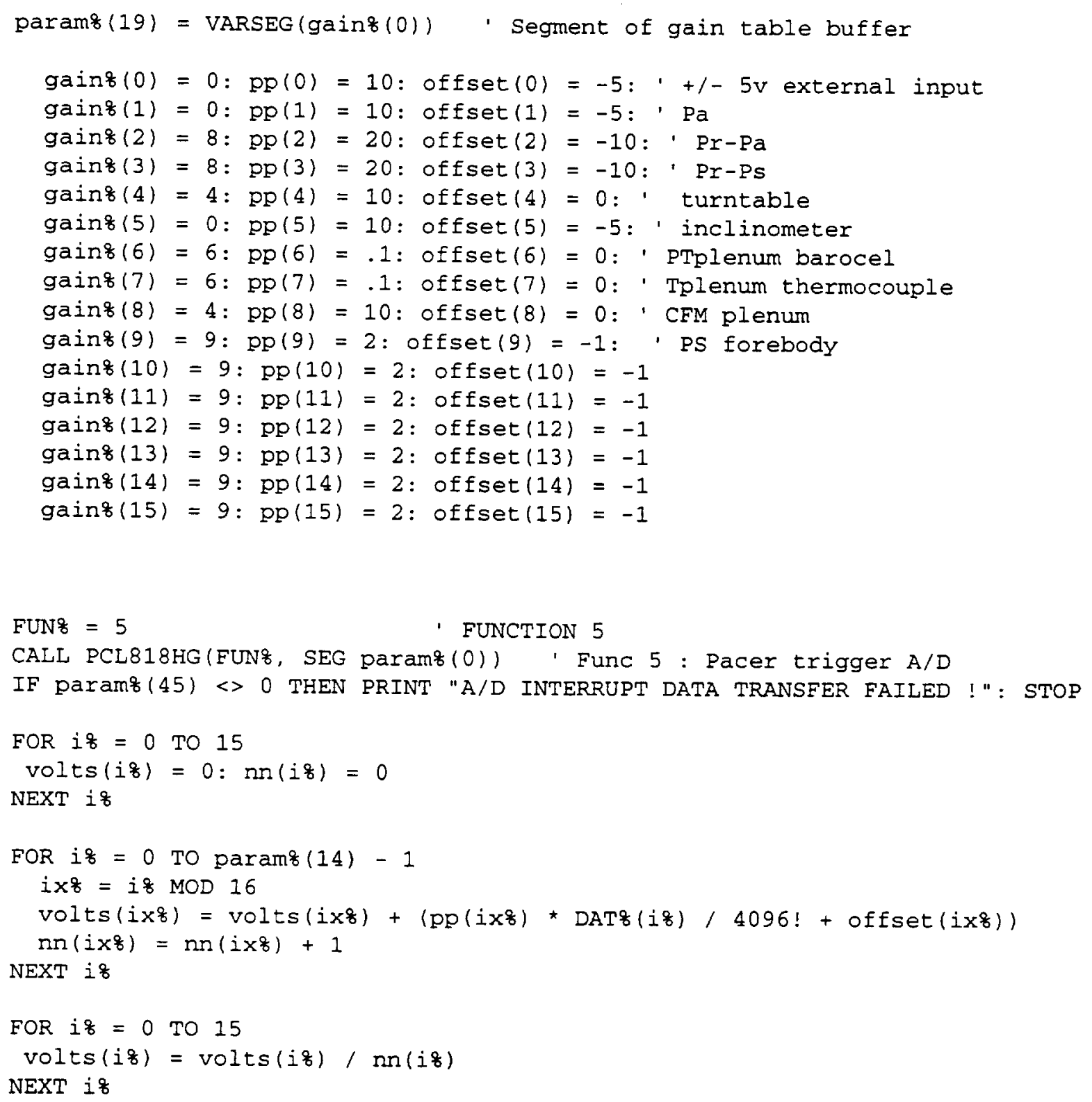




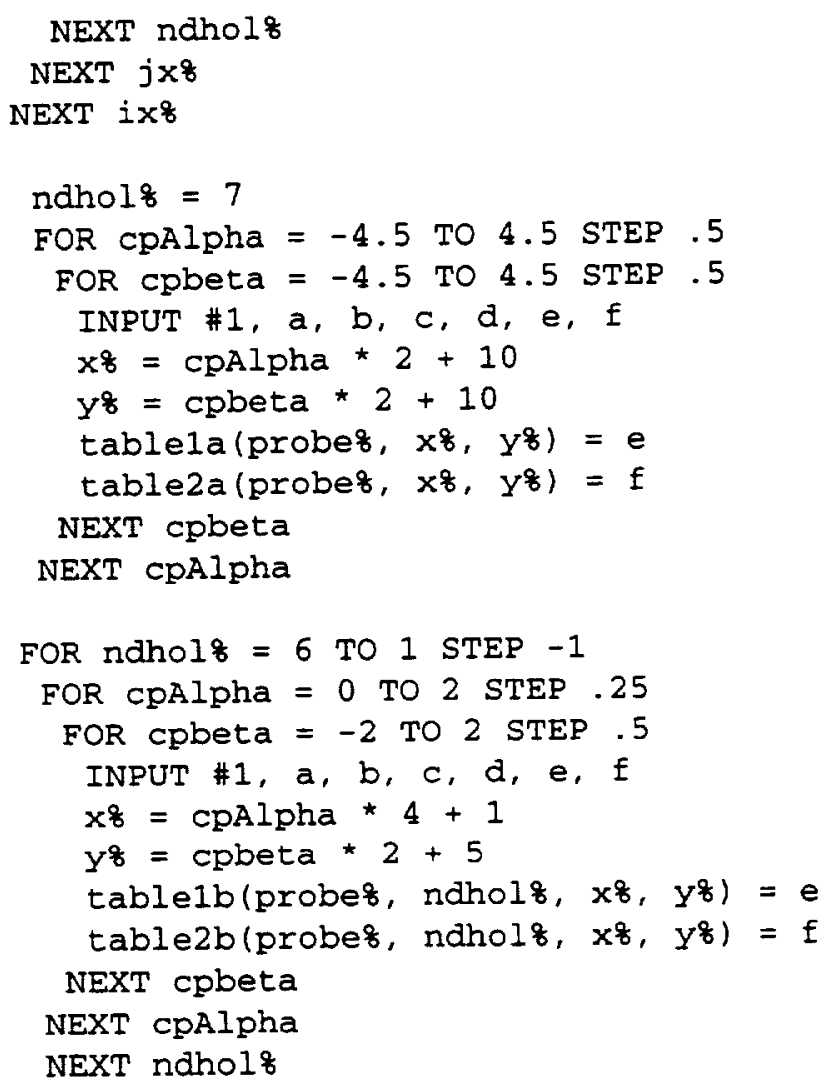




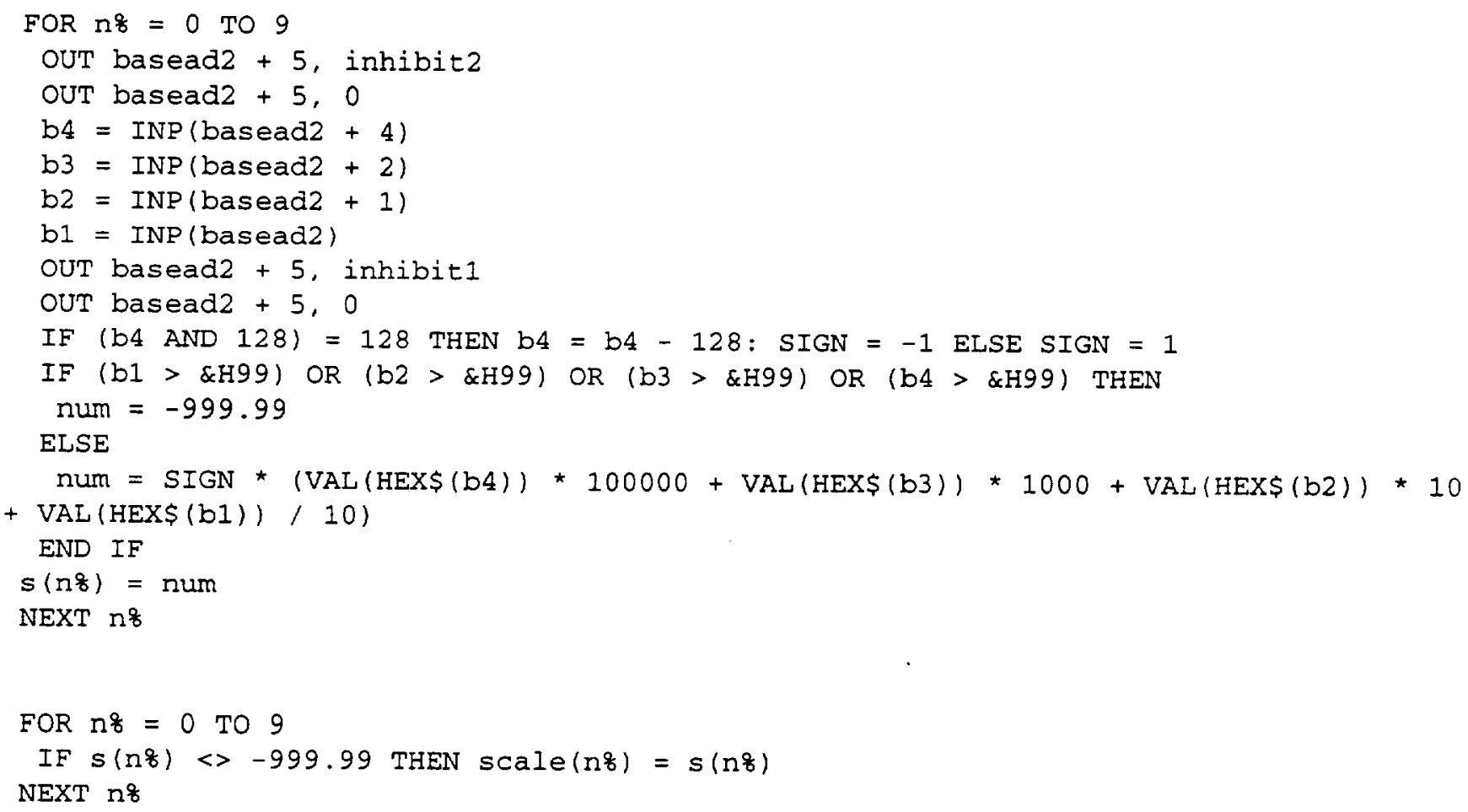




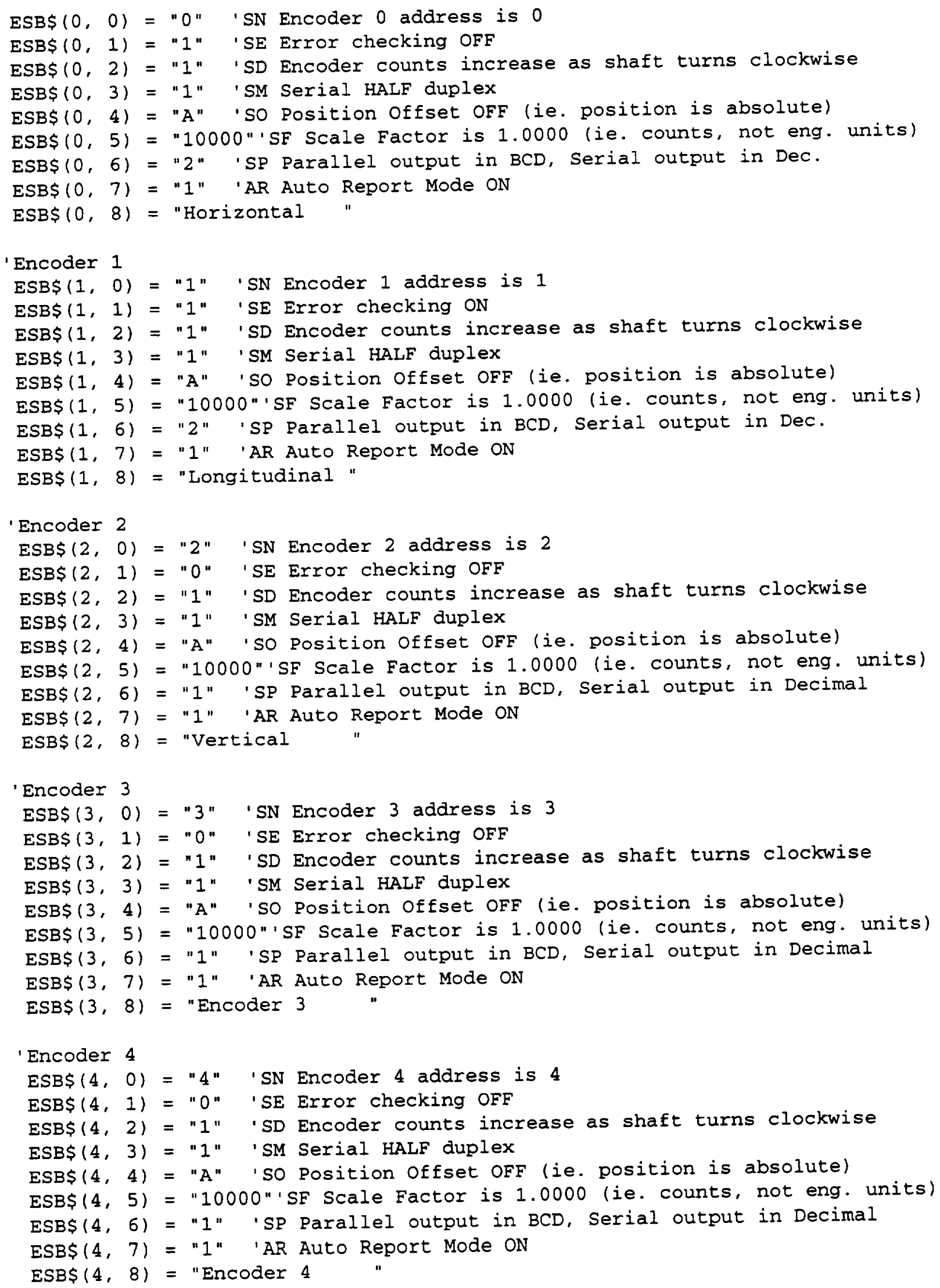




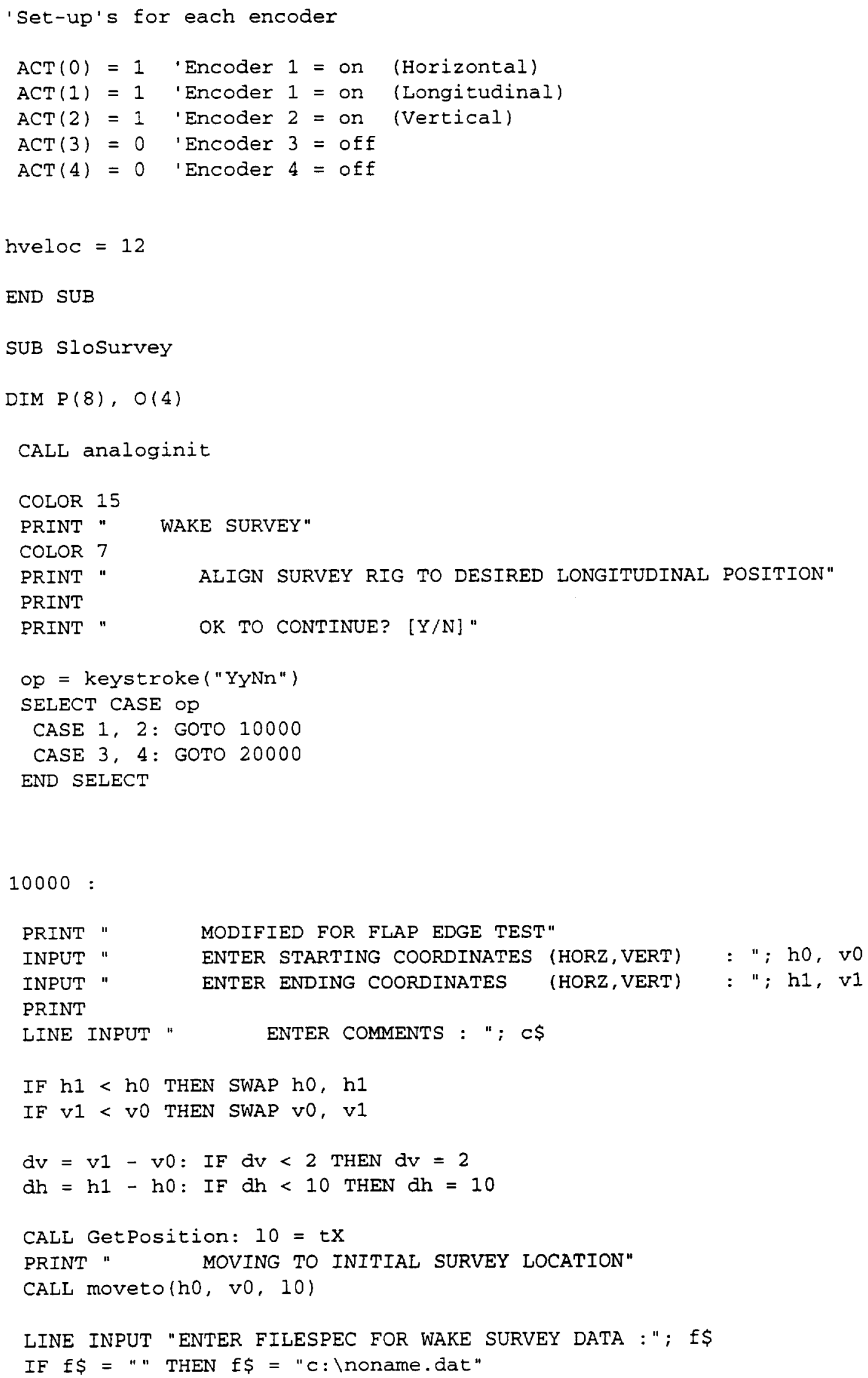




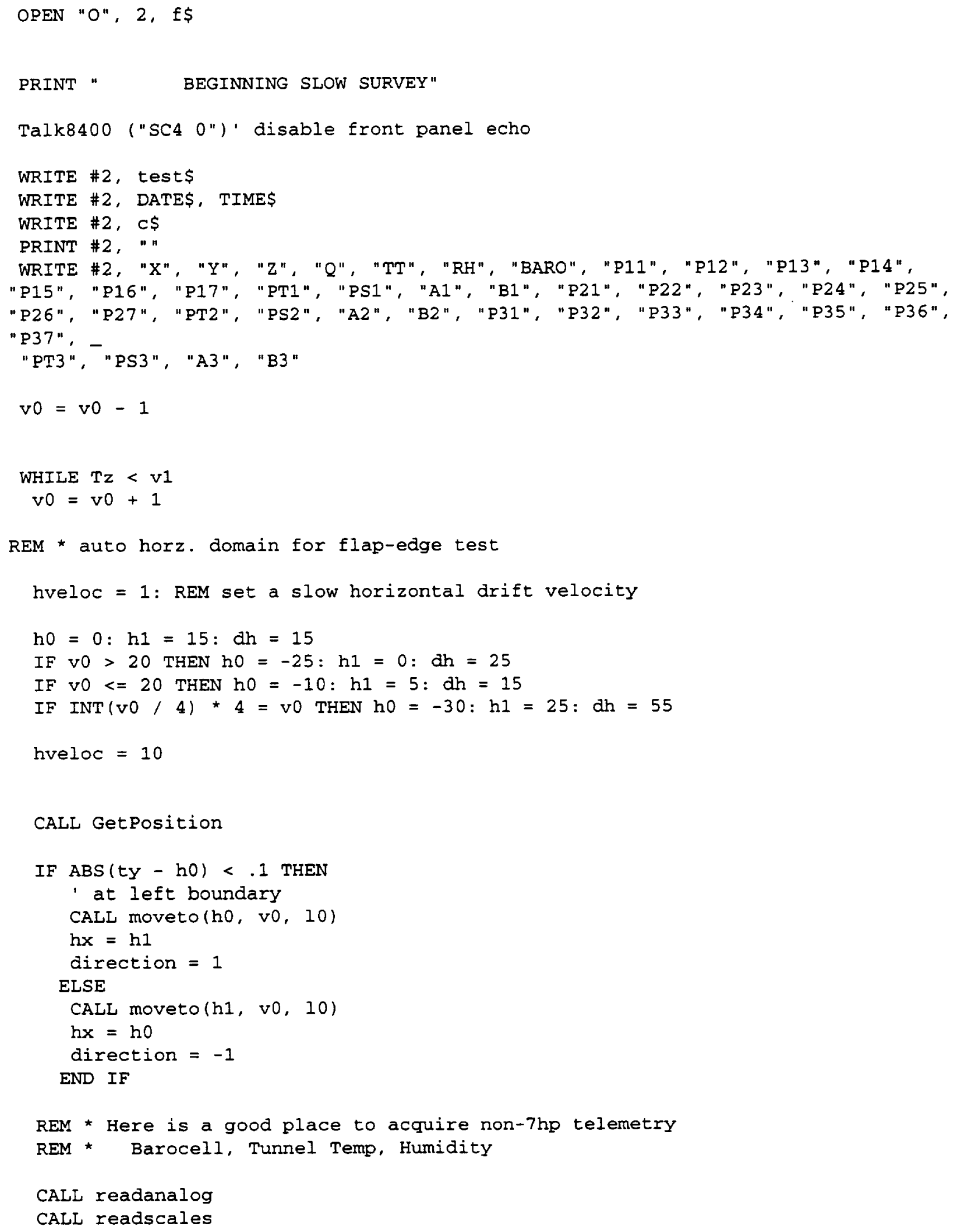




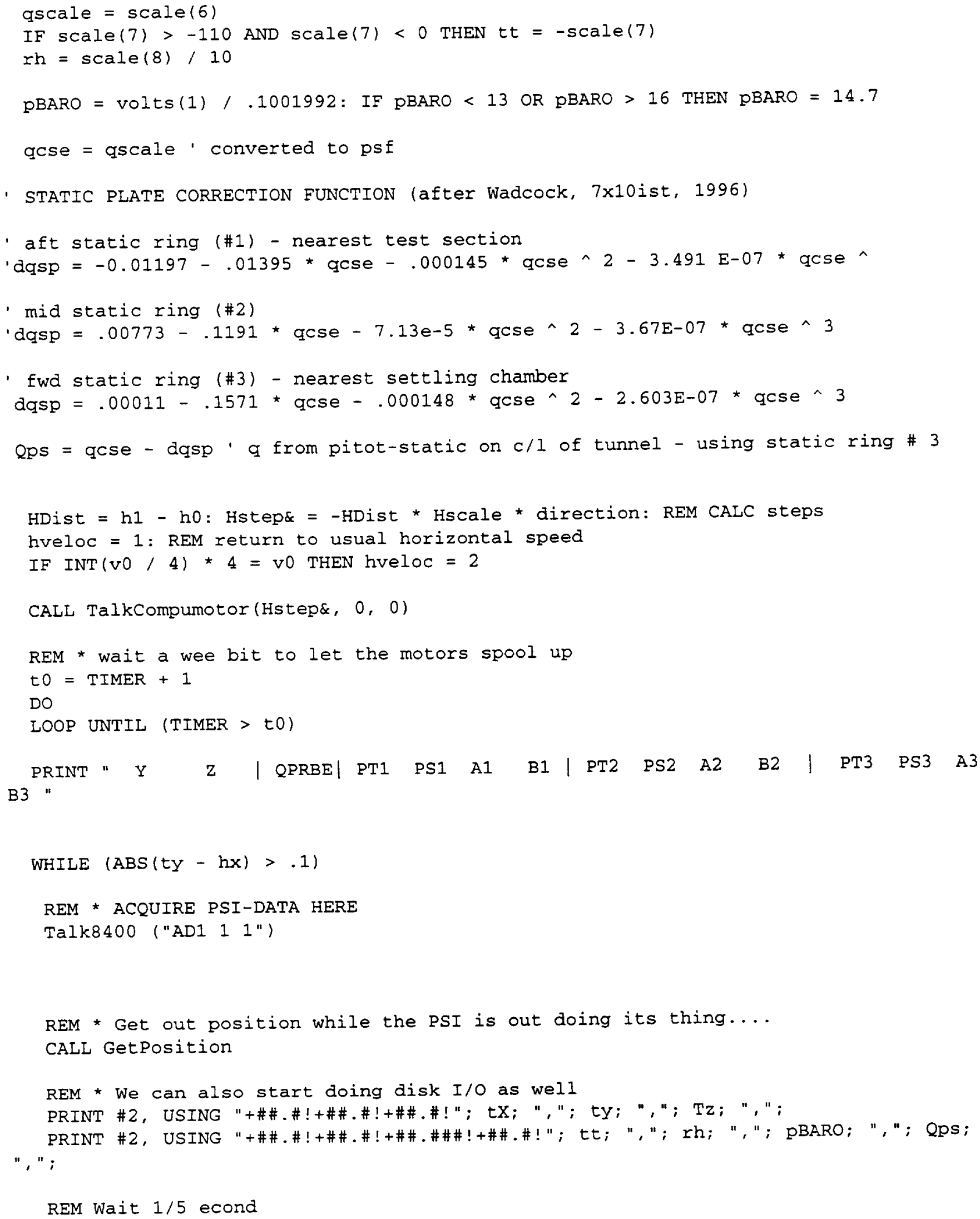




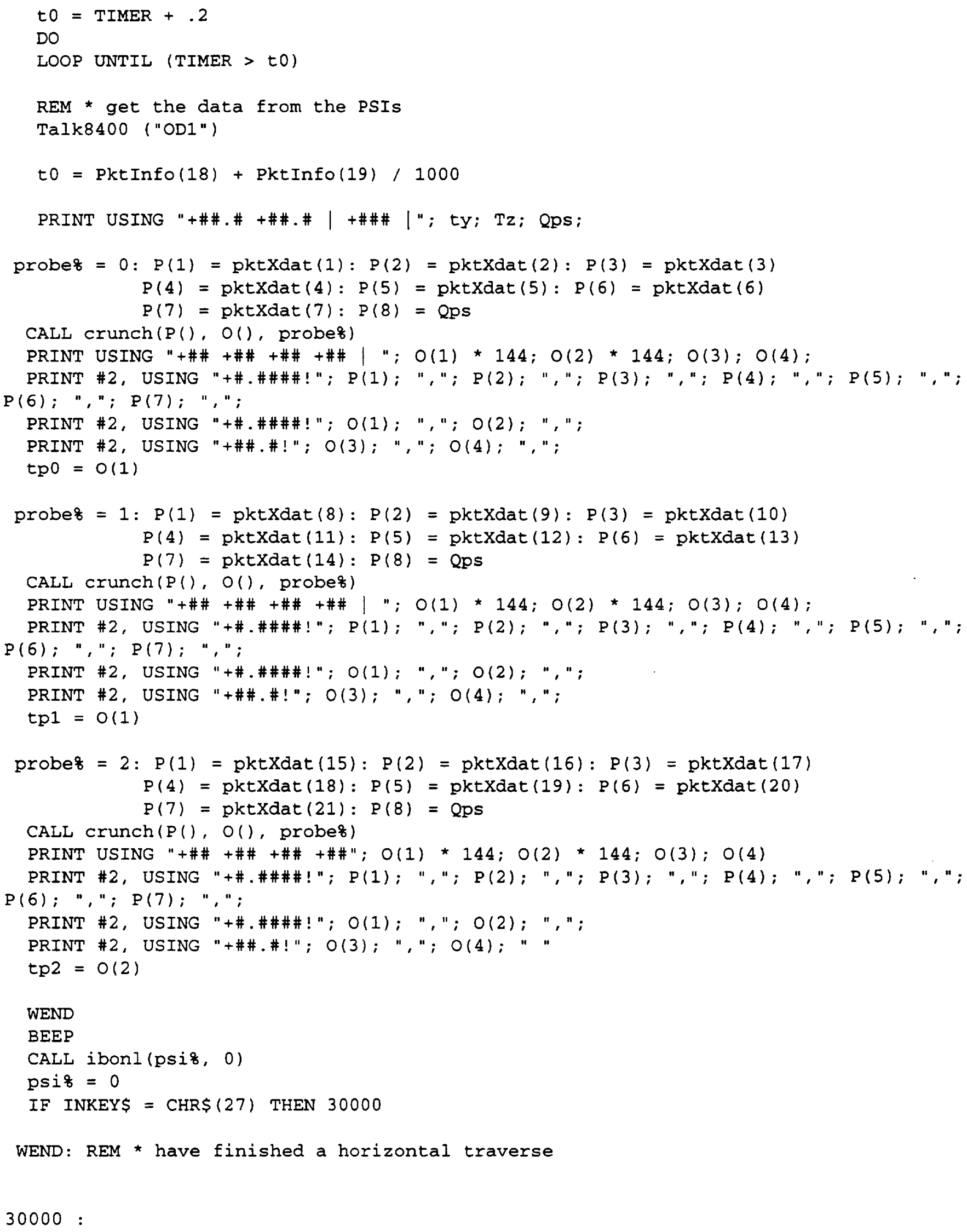




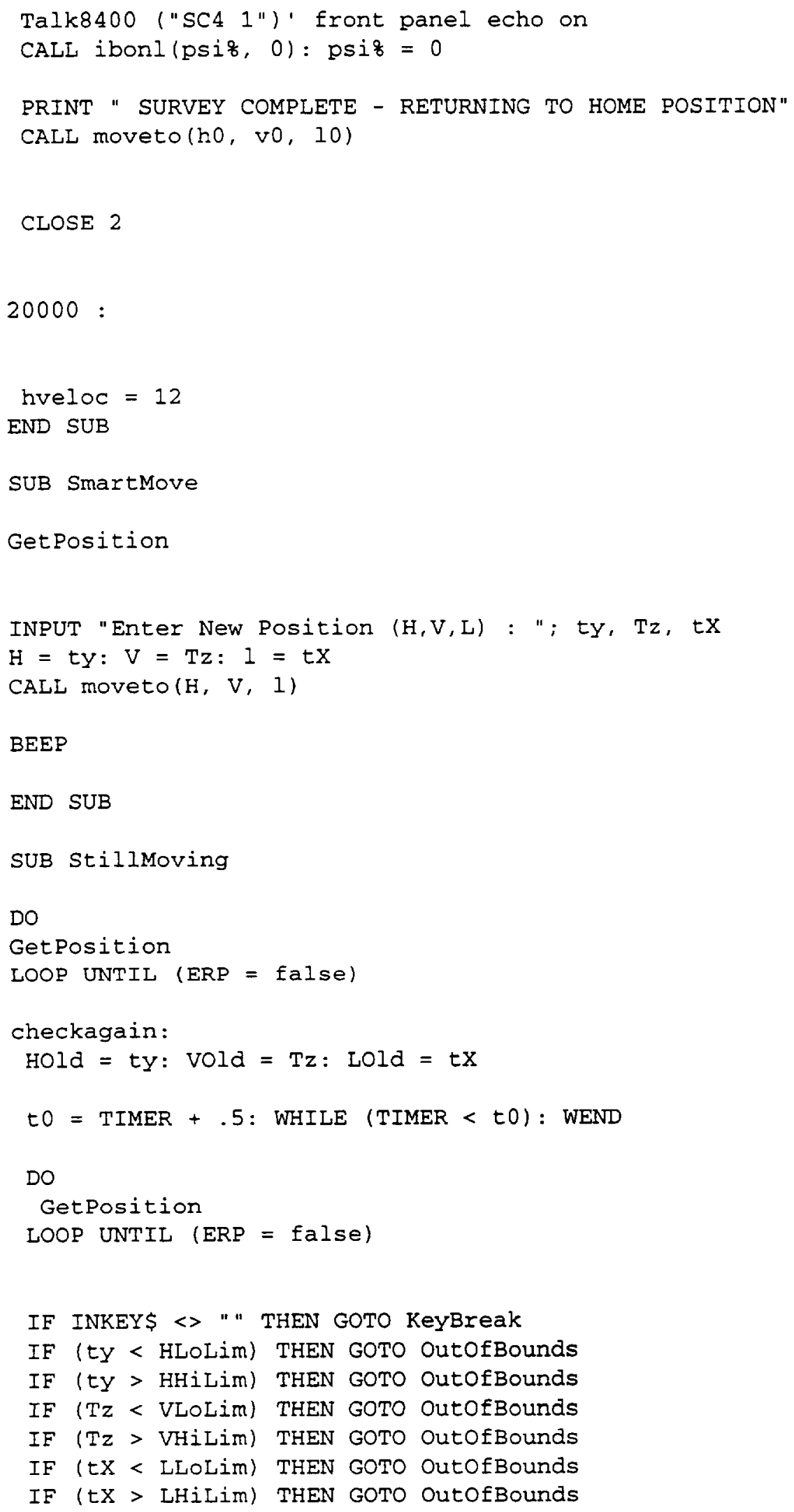




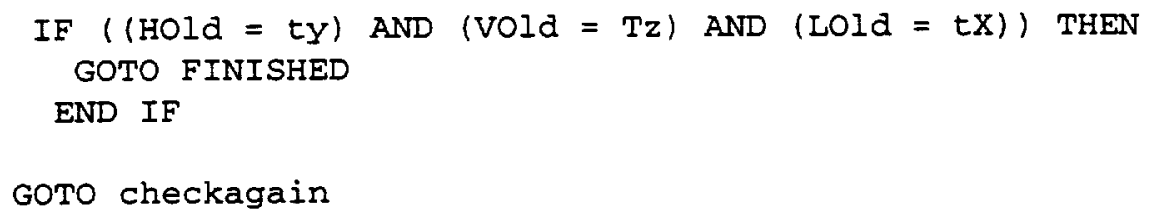




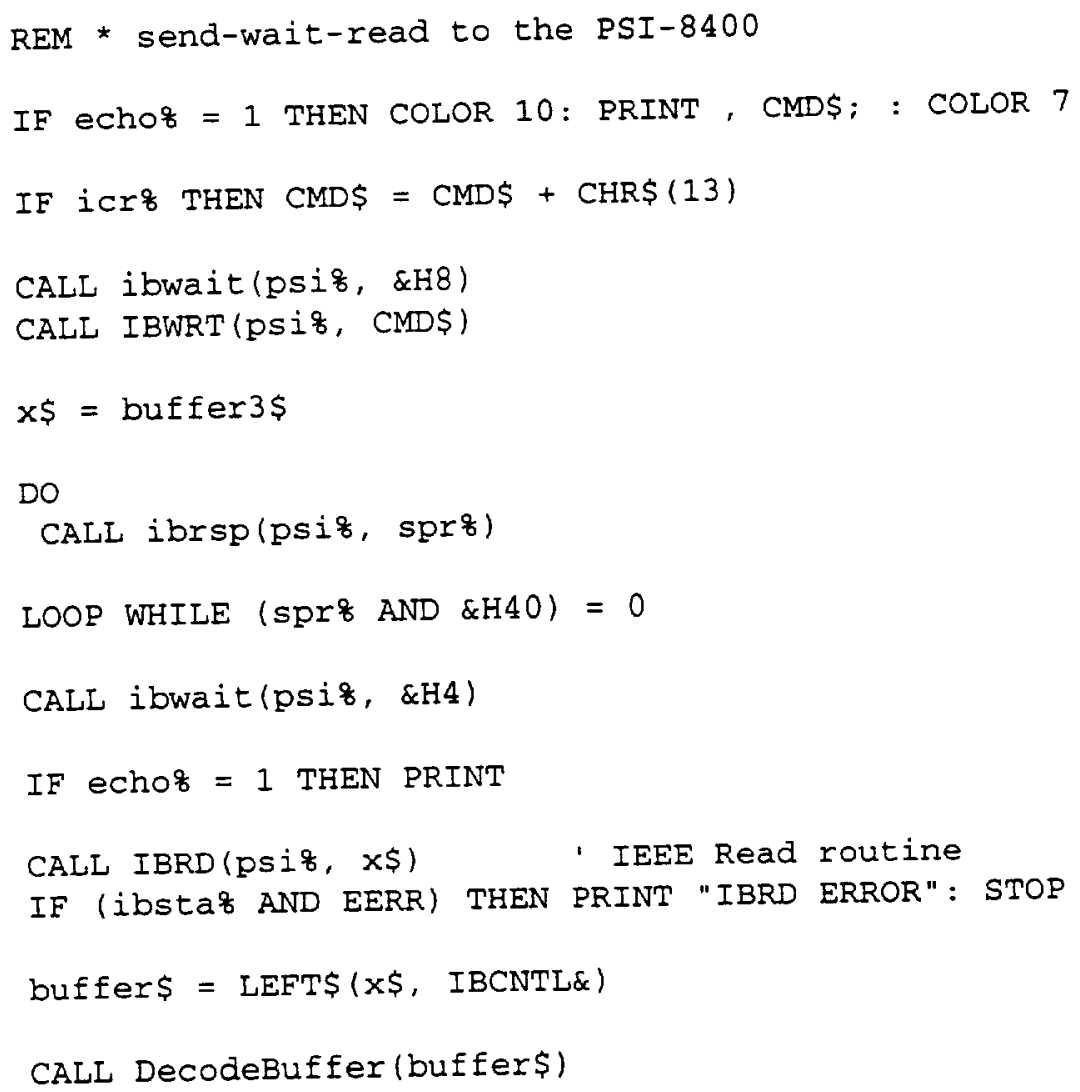


Public reporting burden for this collection of information is estimated to average 1 hour per response, including the time lor reviewing instructions, searching existing data sources. gathering and maintaining the data needed, and completing and reviewing the collection of information. Send comments regarding this burden estimate or any other aspect of this Davis Highway, Suite 1204 . Arlington, Davis Highway. Suite 1204, Arlington, VA 22202-4302, and to the Oflice of Management and Budget, Paperwork Reduction Project (0704-0188), Washington, DC 20503. \begin{tabular}{|l|l|l} 
1. AGENCY USE ONLY (Leave blank) & $\begin{array}{c}\text { 2. REPORT DATE } \\
\text { May } 1997\end{array}$ & $\begin{array}{c}\text { 3. AEPORT TYPE AND DATES COVERED } \\
\text { Technical Memorandum }\end{array}$
\end{tabular}

4. TITLE AND SUBTITLE

Measurement of Air Flow Characteristics Using Seven-Hole Cone Probes

6. AUTHOR(S)

$505-59-53$

Timothy T. Takahashi

7. PERFORMING ORGANIZATION NAME(S) AND ADDRESS(ES)

8. PERFORMING ORGANIZATION REPORT NUMBER

Ames Research Center

Moffett Field, CA 94035-1000

A-976463A

9. SPONSORING/MONITORING AGENCY NAME(S) AND ADDRESS(ES)

10. SPONSORING/MONITORING AGENCY REPORT NUMBER

National Aeronautics and Space Administration

Washington, DC 20546-0001

NASA TM-112194

11. SUPPLEMENTARY NOTES

Point of Contact: Timothy T. Takahashi, Ames Research Center, MS 247-2, Moffett Field, CA 94035-1000 (415) $604-4976$

12a. DISTRIBUTION/AVAILABILITY STATEMENT

Unclassified - Unlimited

Subject Category 09,35

\section{ABSTRACT (Maximum 200 words)}

The motivation for this work has been the development of a wake survey system. A seven-hole probe can measure the distribution of static pressure, total pressure, and flow angularity in a wind tunnel environment. The author describes the development of a simple, very efficient algorithm to compute flow properties from probe tip pressures. Its accuracy and applicability to unsteady, turbulent flow are discussed.

14. SUBJECT TERMS

Instrumentation, Pressure, Flow angle, Flow velocity

12b. DISTRIBUTION CODE 